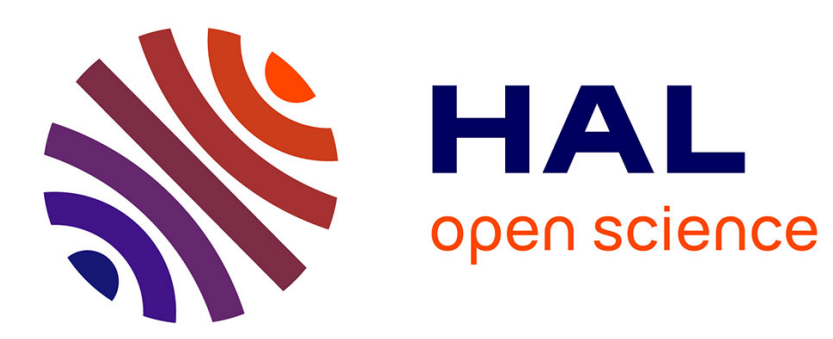

\title{
A numerical scheme for a kinetic model for mixtures in the diffusive limit using the moment method
}

\author{
Andrea Bondesan, Laurent Boudin, Bérénice Grec
}

\section{To cite this version:}

Andrea Bondesan, Laurent Boudin, Bérénice Grec. A numerical scheme for a kinetic model for mixtures in the diffusive limit using the moment method. Numerical Methods for Partial Differential Equations, 2019, 35 (3), pp.1184-1205. 10.1002/num.22345 . hal-01727725

\section{HAL Id: hal-01727725 \\ https://hal.science/hal-01727725}

Submitted on 9 Mar 2018

HAL is a multi-disciplinary open access archive for the deposit and dissemination of scientific research documents, whether they are published or not. The documents may come from teaching and research institutions in France or abroad, or from public or private research centers.
L'archive ouverte pluridisciplinaire HAL, est destinée au dépôt et à la diffusion de documents scientifiques de niveau recherche, publiés ou non, émanant des établissements d'enseignement et de recherche français ou étrangers, des laboratoires publics ou privés. 


\title{
A NUMERICAL SCHEME FOR A KINETIC MODEL FOR MIXTURES IN THE DIFFUSIVE LIMIT USING THE MOMENT METHOD
}

\author{
ANDREA BONDESAN, LAURENT BOUDIN, AND BÉRÉNICE GREC
}

\begin{abstract}
In this article, we consider a multi-species kinetic model which leads to the MaxwellStefan equations under a standard diffusive scaling (small Knudsen and Mach numbers). We propose a suitable numerical scheme which approximates both the solution of the kinetic model in rarefied regime and the one in the diffusion limit. We prove some a priori estimates (mass conservation and nonnegativity) and well-posedness of the discrete problem. We also present numerical examples where we observe the asymptotic-preserving behavior of the scheme.
\end{abstract}

\section{INTRODUCTION}

The derivation and mathematical analysis of models describing gaseous mixtures raise many questions, from the theoretical as well as from the numerical point of view. A wide variety of models can be considered, depending on both the scale and the setting where the model is derived. In this article, we focus on describing mixtures in a diffusive setting at a macroscopic scale. Such models arise in various fields of physics and medicine, for example in the context of respiration, in order to describe the diffusive flow of air in the lower part of the lungs, or for the modelling of polluting particles. The first macroscopic diffusion models for mixtures have been introduced on the one hand by Maxwell and Stefan [38, 44], and by Fick on the other hand [20]. Both models lie in the family of cross-diffusion models [13, 15, 34, 16], coupling the molar fluxes and the mole fractions of each species of the mixture. The Maxwell-Stefan equations have been derived at the macroscopic level from mechanical considerations [36, 41, whereas the Fick equations are obtained from Onsager's contributions from the thermodynamic of irreversible processes. Fick and Maxwell-Stefan formulations have a strong formal analogy, and both models can surely be linked in some regimes [23, 24, 8].

In this work, we shall focus on the Maxwell-Stefan equations, in the setting of a non-reactive mixture of $p \geq 2$ ideal gases in an open bounded subset $\Omega$ of $\mathbb{R}^{d}, d \geq 1$. For any species $\mathcal{A}_{i}, 1 \leq i \leq p$, we denote by $c_{i}$ its concentration in the mixture and $u_{i}$ the associated velocity, which both depend on time $t \geq 0$ and position $x \in \Omega$. Mass conservation is written in $\mathbb{R}^{+} \times \Omega$, for any $1 \leq i \leq p$, as

$$
\partial_{t} c_{i}+\nabla_{x} \cdot\left(c_{i} u_{i}\right)=0 \text {. }
$$

Now, defining the total concentration $c=\sum_{i} c_{i}$, the Maxwell-Stefan diffusion equations are written in $\mathbb{R}^{+} \times \Omega$, for any $1 \leq i \leq p$,

$$
-\nabla_{x}\left(\frac{c_{i}}{c}\right)=\frac{1}{c} \sum_{k \neq i} \frac{c_{k} c_{i} u_{i}-c_{i} c_{k} u_{k}}{c D_{i k}},
$$

where $D_{i k}=D_{k i}>0$ is the binary diffusion coefficient between species $\mathcal{A}_{i}$ and $\mathcal{A}_{k}$. The model is supplied with initial conditions $c_{i}(0, \cdot)=c_{i}^{\text {in }}, u_{i}(0, \cdot)=u_{i}^{\text {in }}$ and boundary conditions on the fluxes $\left.u_{i} \cdot \boldsymbol{n}\right|_{\partial \Omega}=0$, where $\boldsymbol{n}$ denotes the exterior normal to the boundary.

Because of the symmetry of the binary diffusion coefficients, the $p$ equations 22) are not linearly independent. Therefore, an additional relationship is needed in order to close the system, for example

This work was partially funded by the French ANR-13-BS01-0004 project Kibord headed by L. Desvillettes. The first and third authors have been partially funded by Université Sorbonne Paris Cité, in the framework of the "Investissements d'Avenir", convention ANR-11-IDEX-0005. 
the equimolar diffusion assumption, which means that the total flux of the mixture $\sum_{i} c_{i} u_{i}$ equals zero for any time and anywhere in $\Omega$. In this equimolar diffusion setting, if, in addition, the total concentration $c$ of the mixture is uniform in space at initial time, it remains constant for all time, and we can assume $c$ to be equal to 1 without loss of generality. Thus, assuming $c(t, x)=1$ for any $(t, x) \in \mathbb{R}^{+} \times \Omega$, equations (2) become, for any $1 \leq i \leq p$,

$$
-\nabla_{x} c_{i}=\sum_{k \neq i} \frac{c_{k} c_{i} u_{i}-c_{i} c_{k} u_{k}}{D_{i k}}
$$

The Maxwell-Stefan equations have been studied recently from both mathematical [24, 10, 8, 35] and numerical [23] viewpoints (see also the review [36]).

A natural question is to justify such macroscopic diffusion models from a kinetic description at the mesoscopic level, mainly the Boltzmann equation. This falls into the wide literature about hydrodynamic limits, beginning with the pioneering works of Bardos, Golse and Levermore [3, 4], which lead to other works on the Navier-Stokes or Euler hydrodynamic limit (see [14, 25, 26, 27, 28, 45]) for a single monoatomic gas.

For mixtures, suitable kinetic models have been introduced in the middle of the 20th century 43 , [40]. Further, consistent BGK-models for mixtures were derived [22, 2, 12, and a generalization of Boltzmann equations for mixtures was introduced in [17] for polyatomic gases with chemical reactions.

For our study, consider a non-reactive mixture of $p$ monoatomic gases, where each species $\mathcal{A}_{i}$ is described by its distribution function $f_{i}$ depending on time $t \geq 0$, position $x \in \Omega$ and velocity $v \in \mathbb{R}^{d}$. Assuming the only interactions between molecules at the microscopic level to be elastic collisions, two molecules of species $\mathcal{A}_{i}$ and $\mathcal{A}_{k}, 1 \leq i, k \leq p$, of respective masses $m_{i}, m_{k}$ and post-collisional velocities $v$ and $v_{*}$, have pre-collisional velocities $v^{\prime}$ and $v_{*}^{\prime}$ given by

$$
\left\{\begin{array}{l}
v^{\prime}=\frac{1}{m_{i}+m_{k}}\left(m_{i} v+m_{k} v_{*}+m_{k}\left|v-v_{*}\right| \sigma\right), \\
v_{*}^{\prime}=\frac{1}{m_{i}+m_{k}}\left(m_{i} v+m_{k} v_{*}-m_{i}\left|v-v_{*}\right| \sigma\right),
\end{array}\right.
$$

where $\sigma \in \mathbb{S}^{d-1}$, because of momentum and energy conservations. The Boltzmann collision operator is then defined, for $v \in \mathbb{R}^{d}$, by

$$
Q_{i k}\left(f_{i}, f_{k}\right)(v)=\int_{\mathbb{R}^{d}} \int_{\mathbb{S}^{d-1}} \mathcal{B}_{i k}\left(v, v_{*}, \sigma\right)\left[f_{i}\left(v^{\prime}\right) f_{k}\left(v_{*}^{\prime}\right)-f_{i}(v) f_{k}\left(v_{*}\right)\right] \mathrm{d} \sigma \mathrm{d} v_{*},
$$

with cross sections $\mathcal{B}_{i k}=\mathcal{B}_{k i}>0$. We restrict our study to Maxwellian molecules, meaning that for any $1 \leq i, k \leq p$, there exist even functions $b_{i k}:[-1,1] \rightarrow \mathbb{R}^{+} \in L^{1}(-1,1)$ such that the cross sections $B_{i k}$ are written

$$
\mathcal{B}_{i k}\left(v, v_{*}, \sigma\right)=b_{i k}\left(\frac{v-v_{*}}{\left|v-v_{*}\right|} \cdot \sigma\right)=b_{i k}(\cos \theta),
$$

where $\theta \in[0, \pi]$ is the deviation angle between $v-v_{*}$ and $\sigma$.

This collision operator satisfies conservation properties [17]

$$
\begin{array}{ll}
\int_{\mathbb{R}^{d}} Q_{i k}\left(f_{i}, f_{k}\right)(v) m_{i} \mathrm{~d} v=0, & 1 \leq i, k \leq p, \\
\int_{\mathbb{R}^{d}} Q_{i i}\left(f_{i}, f_{i}\right)(v) m_{i} v \mathrm{~d} v=0, & 1 \leq i \leq p .
\end{array}
$$

The Boltzmann equations for mixtures are then written, for $1 \leq i \leq p$,

$$
\partial_{t} f_{i}+v \cdot \nabla_{x} f_{i}=\sum_{k=1}^{p} Q_{i k}\left(f_{i}, f_{k}\right), \quad \text { on } \mathbb{R}^{+} \times \Omega \times \mathbb{R}^{d}
$$


In the context of mixtures, formal hydrodynamical limits have been performed in several regimes. We here focus on the diffusive scaling (small Knudsen and Mach numbers). Euler and Navier-Stokes equations, coupled or not with a Vlasov equation, were obtained as the fluid-dynamic limit of the Boltzmann equations for a binary mixture [5, 6], as well as the incompressible Euler and NavierStokes equations for general mixtures [7]. It has also been shown in [11] that Maxwell-Stefan's equations can be seen as the limit in the small Mach and Knudsen number regime of the Boltzmann equations for mixtures in the case of Maxwellian molecules. This result has been extended to some analytical cross sections in [30, and generalized to general cross sections in [9], as well as in a non-isothermal setting in [29].

In this article, we are concerned with the same diffusive scaling as in [11, assuming Mach and Knudsen numbers to be of the same order of magnitude $\varepsilon \ll 1$. Then equations (6) are written, for $1 \leq i \leq p$,

$$
\varepsilon \partial_{t} f_{i}^{\varepsilon}+v \cdot \nabla_{x} f_{i}^{\varepsilon}=\frac{1}{\varepsilon} \sum_{k=1}^{p} Q_{i k}\left(f_{i}^{\varepsilon}, f_{k}^{\varepsilon}\right), \quad \text { on } \mathbb{R}^{+} \times \Omega \times \mathbb{R}^{d} .
$$

Their formal hydrodynamic limit obtained thanks to a moment method are the Maxwell-Stefan equations (3) on $c_{i}$ and $c_{i} u_{i}, 1 \leq i \leq p, c_{i}$ and $c_{i} u_{i}$ being the limits, when $\varepsilon$ vanishes, of the zerothand first-order moments of the distribution functions $f_{i}^{\varepsilon}, 1 \leq i \leq p$.

We here aim to write a numerical scheme which could both capture the behavior of physical solutions to the Boltzmann equations in a rarefied regime and describe the solutions of the Maxwell-Stefan equations in the fluid regime. However, this induces some difficulties, due to the fact that the collision term (and, in a lesser way, the transport term) becomes stiffer when the parameter $\varepsilon$ tends to zero. In particular, a satisfactory numerical scheme needs to use time and space steps independent of the parameter $\varepsilon$, which falls into the class of asymptotic-preserving (AP) schemes. Such AP schemes have been derived for many equations in several regimes [21], and we refer the reader to the review [31] treating AP schemes for kinetic and hyperbolic equations. In the context of kinetic equations for mixtures, AP schemes capturing the Euler and Navier-Stokes hydrodynamic limit from the Boltzmann equation for mixtures are derived in [33, 32].

In the following, in order to describe numerical schemes, let us introduce a time discretization $\left(t^{n}\right)_{n \in \mathbb{N}}$ with a time step $\Delta t>0$, such that $t^{n}=n \Delta t$.

A reasonable attempt to derive an AP numerical scheme for Boltzmann equations for mixtures is to use a penalty method by a linear BGK-operator as in [21, which has been extended for mixtures in [32]. This operator would then be defined for each species $1 \leq i \leq p$ as $P_{i}: f_{i} \mapsto \beta_{i}\left(M_{i}-f_{i}\right)$, where $M_{i}$ is the global Maxwellian equilibrium state defined by

$$
M_{i}(t, x, v)=c_{i}(t, x)\left(\frac{m_{i}}{2 \pi k_{B} T}\right)^{1 / 2} \exp \left(-\frac{m_{i} v^{2}}{2 k_{B} T}\right),
$$

and $\beta_{i}$ is some constant to be specified. The semi-discretized scheme is then written, for $1 \leq i \leq p$,

$$
\frac{f_{i}^{n+1}-f_{i}^{n}}{\Delta t}+\frac{1}{\varepsilon} v \cdot \nabla_{x} f_{i}^{n}=\frac{Q_{i}^{n}-P_{i}^{n}}{\varepsilon^{2}}+\frac{P_{i}^{n+1}}{\varepsilon^{2}},
$$

where $f_{i}^{n} \simeq f_{i}^{\varepsilon}\left(t^{n}, x, v\right), P_{i}^{n}=P_{i}\left(f_{i}^{n}\right)$, and $Q_{i}^{n}=\sum_{k=1}^{p} Q_{i k}\left(f_{i}^{n}, f_{k}^{n}\right)$.

However, such a scheme leads to a transport term of order $1 / \varepsilon$, which imposes a very fine discretization for the velocity variable, and a growing support in the velocity variable, since the velocity domain is unbounded. Therefore, we had to choose another method.

Our approach is to mimic the analytical method used to obtain the Maxwell-Stefan equations in the low Mach and Knudsen numbers limit from the Boltzmann equations for mixtures. Whereas the Fick equations are naturally obtained from the kinetic equations by a perturbative method, the Maxwell-Stefan ones are obtained, as we already stated, by a moment method [9]. Thus the scheme 
we propose relies on the computation of the moments of the distribution functions $f_{i}^{\varepsilon}, 1 \leq i \leq p$, under the ansatz that these distributions functions are at local equilibria states. In this way, we can derive a one-dimensional (we choose $d=1$ ) numerical scheme for the Boltzmann kinetic model for mixtures, which nicely converges to the Maxwell-Stefan equations in the small Mach and Knudsen numbers limit.

The paper is organized as follows. In Section 2, we briefly recall the moment method for the Boltzmann equations for mixtures and describe the chosen numerical scheme. A priori estimates for this scheme are proved in Section 3, in particular the positivity of the concentrations. In Section 4. these estimates are used to prove the existence of a solution to the numerical scheme. Last, in Section 5. we present numerical simulations illustrating the asymptotic-preserving behavior of the scheme, and its capacity to describe uphill diffusion phenomena for mixtures.

\section{Derivation of a numerical scheme for Boltzmann EQUations For miXtures}

Consider also a space discretization $\left(x_{j}\right)_{0 \leq j \leq N}$ of the domain $\Omega$, with a space step $\Delta x>0$, such that $x_{j}=j \Delta x$.

2.1. Moment method. Since formal theoretical asymptotic results [11, 9] are obtained by a moment method [37], assuming that the distribution function of each species $i$ is at a local equilibrium state with a small velocity for any $(t, x) \in \mathbb{R}^{+} \times \Omega$, we apply the same approach in order to derive a numerical scheme which nicely behaves when $\varepsilon$ tends to 0 . More precisely, the results are obtained under the following ansatz, for any $1 \leq i \leq p$,

$$
f_{i}^{\varepsilon}(t, x, v)=c_{i}^{\varepsilon}(t, x)\left(\frac{m_{i}}{2 \pi k_{B} T}\right)^{1 / 2} \exp \left(-\frac{m_{i}\left(v-\varepsilon u_{i}^{\varepsilon}(t, x)\right)^{2}}{2 k_{B} T}\right), \quad \forall(t, x, v) \in \mathbb{R}^{+} \times \Omega \times \mathbb{R} .
$$

The same ansatz is also made on the the initial condition $f_{i}^{\text {in }}(x, v)=f_{i}^{\varepsilon}(0, x, v)$. In order for the scheme to be consistent with the possible closure relation of equimolar diffusion, we assume that the macroscopic quantities $c_{i}^{\text {in }}(x)=c_{i}(0, x)$ and $u_{i}^{\text {in }}(x)=u_{i}(0, x)$ satisfy the compatibility conditions

$$
\sum_{i=1}^{p} c_{i}^{\text {in }}=1 \text { and } \sum_{i=1}^{p} c_{i}^{\text {in }} u_{i}^{\text {in }}=0
$$

When integrating with respect to $v$ equations (7) and using (4), we obtain, for $1 \leq i \leq p$,

$$
\varepsilon \partial_{t} c_{i}^{\varepsilon}+\int_{\mathbb{R}} v \partial_{x} f_{i}^{\varepsilon} \mathrm{d} v=0
$$

which, using ansatz (8), becomes the usual mass conservation for any species $1 \leq i \leq p$

$$
\partial_{t} c_{i}^{\varepsilon}+\partial_{x}\left(c_{i}^{\varepsilon} u_{i}^{\varepsilon}\right)=0
$$

Now, we integrate with respect to $v$ equations (7) multiplied by $m_{i} v$ to obtain, for any $1 \leq i \leq p$

$$
\varepsilon^{2} m_{i} \partial_{t}\left(c_{i}^{\varepsilon} u_{i}^{\varepsilon}\right)+m_{i} \int_{\mathbb{R}} v^{2} \partial_{x} f_{i}^{\varepsilon} \mathrm{d} v=\frac{m_{i}}{\varepsilon} \sum_{k=1}^{p} \int_{\mathbb{R}} Q_{i k}\left(f_{i}^{\varepsilon}, f_{k}^{\varepsilon}\right) v \mathrm{~d} v
$$

which becomes, using (5) and again ansatz (8) as in [11,

$$
\varepsilon^{2} m_{i} \partial_{t}\left(c_{i}^{\varepsilon} u_{i}^{\varepsilon}\right)+\varepsilon^{2} m_{i} \partial_{x}\left(c_{i}^{\varepsilon}\left(u_{i}^{\varepsilon}\right)^{2}\right)+k_{B} T \partial_{x} c_{i}^{\varepsilon}=\sum_{k \neq i} \mu_{i k} B_{i k} c_{i} c_{k}\left(u_{k}-u_{i}\right)
$$

where $\mu_{i k}=\frac{2 m_{i} m_{k}}{m_{i}+m_{k}}$ is the reduced mass corresponding to species $\mathcal{A}_{i}$ and $\mathcal{A}_{k}$, and $B_{i k}=\frac{1}{2}\left\|b_{i k}\right\|_{L^{1}(-1,1)}>$ 0 is the constant cross-section for Maxwellian molecules in dimension 1. This equation can be written 
in a matrix form introducing the coefficients $\left(A_{i k}^{\varepsilon}\right)_{1 \leq i, k \leq p}$

$$
A_{i k}^{\varepsilon}= \begin{cases}-\mu_{i k} B_{i k} c_{i}^{\varepsilon}, & \text { if } i \neq k, \\ \sum_{\ell \neq i} \mu_{i \ell} B_{i \ell} c_{\ell}^{\varepsilon}, & \text { if } i=k .\end{cases}
$$

Let us denote $\mathcal{F}^{\varepsilon}=\left(c_{i}^{\varepsilon} u_{i}^{\varepsilon}\right)_{1 \leq i \leq p}$ the column vector of the fluxes, and $\left[A^{\varepsilon} \mathcal{F}^{\varepsilon}\right]_{i}=\sum_{k=1}^{p} A_{i k}^{\varepsilon} c_{k}^{\varepsilon} u_{k}^{\varepsilon}$ the $i$-th coordinate of the product vector $A^{\varepsilon} \mathcal{F}^{\varepsilon}$. Equation $(11)$ is then written

$$
\varepsilon^{2} m_{i} \partial_{t}\left(c_{i}^{\varepsilon} u_{i}^{\varepsilon}\right)+\varepsilon^{2} m_{i} \partial_{x}\left(c_{i}^{\varepsilon}\left(u_{i}^{\varepsilon}\right)^{2}\right)+k_{B} T \partial_{x} c_{i}^{\varepsilon}=-\left[A^{\varepsilon} \mathcal{F}^{\varepsilon}\right]_{i} .
$$

Now, denoting $F_{i}^{\varepsilon}=c_{i}^{\varepsilon} u_{i}^{\varepsilon}$, we obtain the following system

$$
\left\{\begin{array}{l}
\partial_{t} c_{i}^{\varepsilon}+\partial_{x} F_{i}^{\varepsilon}=0, \\
\varepsilon^{2} m_{i} \partial_{t} F_{i}^{\varepsilon}+\varepsilon^{2} m_{i} \partial_{x}\left(c_{i}^{\varepsilon}\left(u_{i}^{\varepsilon}\right)^{2}\right)+k_{B} T \partial_{x} c_{i}^{\varepsilon}=-\left[A^{\varepsilon} \mathcal{F}^{\varepsilon}\right]_{i} .
\end{array}\right.
$$

This system is supplemented with initial conditions on the concentrations and fluxes, as well as boundary conditions on the fluxes, for any $1 \leq i \leq p$

$$
\begin{aligned}
& c_{i}^{\varepsilon}(0, \cdot)=c_{i}^{\text {in }} \geq 0, \text { and } F_{i}^{\varepsilon}(0, \cdot)=F_{i}^{\text {in }}, \quad \text { in } \Omega, \\
& F_{i}^{\varepsilon}(t, \cdot)_{\mid \partial \Omega}=0, \quad \forall t>0 .
\end{aligned}
$$

2.2. Description of the numerical scheme. The scheme is obtained thanks to a discretization of system (14) using a staggered dual grid. For each species $1 \leq i \leq p$, its concentration $c_{i}$ is evaluated at the points $x_{j}, 0 \leq j \leq N$, whereas its flux $F_{i}$ is evaluated at $x_{j+\frac{1}{2}}=\left(j+\frac{1}{2}\right) \Delta x$, for $0 \leq j \leq N-1$. Therefore, we shall denote $c_{i, j}^{n} \simeq c_{i}^{\varepsilon}\left(t^{n}, x_{j}\right)$, and $F_{i, j+\frac{1}{2}}^{n} \simeq F_{i}^{\varepsilon}\left(t^{n}, x_{j+\frac{1}{2}}\right)$ the numerical approximations of the unknowns at the discretization points. Introducing $\lambda=\Delta t / \Delta x$, the first equation of system (14) can then be discretized as follows, for any $1 \leq i \leq p$ and $0 \leq j \leq N$

$$
c_{i, j}^{n+1}+\lambda\left(F_{i, j+\frac{1}{2}}^{n+1}-F_{i, j-\frac{1}{2}}^{n+1}\right)=c_{i, j}^{n} .
$$

For the second one, we observe that the matrix $A$ contains concentrations, which have to be evaluated at the same points as the fluxes. In order to prove nonnegativity of the concentrations (Proposition 4), we shall see that a relevant choice [1] for the concentrations is

$$
c_{i, j+\frac{1}{2}}^{n+1}=\min \left\{c_{i, j}^{n+1}, c_{i, j+1}^{n+1}\right\} .
$$

Moreover, we have to discretize the nonlinear term $c_{i} u_{i}^{2}$. Let us introduce the following quantities, for $1 \leq i \leq p$ and $0 \leq j \leq N-1$,

$$
r_{i, j}^{n}= \begin{cases}\frac{1}{4 c_{i, j}^{n}}\left(F_{i, j+\frac{1}{2}}^{n}+F_{i, j-\frac{1}{2}}^{n}\right)^{2} & \text { if } c_{i, j}^{n} \neq 0, \\ 0 & \text { if } c_{i, j}^{n}=0 .\end{cases}
$$

We denote by $R_{i, j+\frac{1}{2}}^{n} / \Delta x$ a discretization of $\partial_{x}\left(c_{i} u_{i}^{2}\right)$ at $x_{j+\frac{1}{2}}$ and time $t^{n}$, chosen such that

$$
R_{i, j+\frac{1}{2}}^{n}=r_{i, j+1}^{n}-r_{i, j}^{n} .
$$

This leads to the following discretization of the second equation of system (14), for any $1 \leq i \leq p$ and $0 \leq j \leq N-1$,

$$
\begin{aligned}
\left(-\Delta t \sum_{k \neq i} \mu_{i k} B_{i k} c_{k, j+\frac{1}{2}}^{n+1}-\varepsilon^{2} m_{i}\right) F_{i, j+\frac{1}{2}}^{n+1} & +\Delta t c_{i, j+\frac{1}{2}}^{n+1} \sum_{k \neq i} \mu_{i k} B_{i k} F_{k, j+\frac{1}{2}}^{n+1} \\
& =k_{B} T \lambda\left(c_{i, j+1}^{n+1}-c_{i, j}^{n+1}\right)+\varepsilon^{2} m_{i} \lambda R_{i, j+\frac{1}{2}}^{n}-\varepsilon^{2} m_{i} F_{i, j+\frac{1}{2}}^{n} .
\end{aligned}
$$


In order for this discretization to be well-defined, we need to supplement it with boundary conditions on the fluxes in ghost cells $F_{i,-\frac{1}{2}}^{n+1}$ and $F_{i, N+\frac{1}{2}}^{n+1}$. In accordance with 16), we choose, for $1 \leq i \leq p$

$$
F_{i,-\frac{1}{2}}^{n+1}=F_{i, N+\frac{1}{2}}^{n+1}=0
$$

which allows the scheme to satisfy mass conservation (Proposition 1). The scheme is thus written, for $1 \leq i \leq p$,

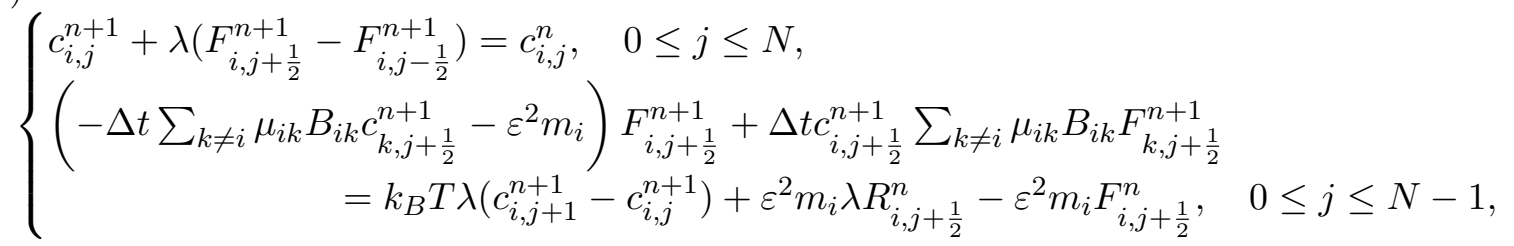

and it can be written for all species simultaneously in a matrix form, introducing the following vector of unknowns $\mathrm{y}^{n}=\left(\begin{array}{l}\mathrm{y}_{1}^{n} \\ \mathrm{y}_{2}^{n}\end{array}\right) \in \mathbb{R}^{p(2 N+1)}$, where

$$
\begin{aligned}
& \mathrm{y}_{1}^{n}=\left(c_{1,0}^{n}, \cdots, c_{1, N}^{n}, \cdots, c_{p, 0}^{n}, \cdots, c_{p, N}^{n}\right)^{\top} \in \mathbb{R}^{p(N+1)}, \\
& \mathrm{y}_{2}^{n}=\left(F_{1, \frac{1}{2}}^{n}, \cdots, F_{1, N-\frac{1}{2}}^{n}, \cdots, F_{p, \frac{1}{2}}^{n}, \cdots, F_{p, N-\frac{1}{2}}^{n}\right)^{\top} \in \mathbb{R}^{p N} .
\end{aligned}
$$

In a similar way, we define

$$
\begin{aligned}
\mathrm{my}_{2}^{n} & =\left(m_{1} F_{1, \frac{1}{2}}^{n}, \cdots, m_{1} F_{1, N-\frac{1}{2}}^{n}, \cdots, m_{p} F_{p, \frac{1}{2}}^{n}, \cdots, m_{p} F_{p, N-\frac{1}{2}}^{n}\right)^{\top} \in \mathbb{R}^{p N}, \\
\mathrm{mR}^{n} & =\left(m_{1} R_{1, \frac{1}{2}}^{n}, \cdots, m_{1} R_{1, N-\frac{1}{2}}^{n}, \cdots, m_{p} R_{p, \frac{1}{2}}^{n}, \cdots, m_{p} R_{p, N-\frac{1}{2}}^{n}\right)^{\top} \in \mathbb{R}^{p N} .
\end{aligned}
$$

Indeed, system (21) then becomes

$$
\mathbb{S}^{\varepsilon}\left(\mathrm{y}_{1}^{n+1}\right) \mathrm{y}^{n+1}=\mathrm{b}^{n}
$$

where

$$
\mathrm{b}^{n}=\left(\mathrm{y}_{1}^{n}, \varepsilon^{2}\left(\lambda \mathrm{mR}^{n}-\mathrm{my}_{2}^{n}\right)\right)^{\top} \in \mathbb{R}^{p(2 N+1)} .
$$

The matrix $\mathbb{S}^{\varepsilon}$ depends on the vector of concentrations $\mathrm{y}_{1}$ and is given by

$$
\mathbb{S}^{\varepsilon}\left(\mathrm{y}_{1}\right)=\left[\begin{array}{cc}
\mathbb{S}_{11} & \mathbb{S}_{12} \\
\mathbb{S}_{21} & \mathbb{S}_{22}^{\varepsilon}\left(\mathrm{y}_{1}\right)
\end{array}\right]
$$

where $\mathbb{S}_{11}=\mathbb{I}_{p(N+1)}$ is the identity matrix of size $p(N+1)$. The other blocks are defined using the following notation.

Notation. For any $q, r, s \in \mathbb{N}^{*}$ and any matrices $M_{\ell} \in \mathbb{R}^{r \times s}, 1 \leq \ell \leq q$, we define the block matrix

$$
\operatorname{Diag}_{q r \times q s}\left(M_{\ell}\right)=\left[\begin{array}{cccc}
M_{1} & & & \\
& M_{2} & & \\
& & \ddots & \\
& & & M_{q}
\end{array}\right] \in \mathbb{R}^{q r \times q s},
$$

where the matrices which do not appear in the block writing are all zero.

The block $\mathbb{S}_{12} \in \mathbb{R}^{p(N+1) \times p N}$ is given by $\mathbb{S}_{12}=\lambda \operatorname{Diag}_{p(N+1) \times p N}\left(S_{12}\right)$. The matrices $\mathbb{S}_{21}$ and $\mathbb{S}_{12}$ are linked by $\mathbb{S}_{21}=k_{B} T \mathbb{S}_{12}^{\top} \in \mathbb{R}^{p N \times p(N+1)}$, and $S_{12} \in \mathbb{R}^{(N+1) \times N}$ is defined by $\left(S_{12}\right)_{i, j}=\delta_{i j}-\delta_{i, j+1}$, 
$1 \leq i \leq N+1,1 \leq j \leq N$. The last block $\mathbb{S}_{22}^{\varepsilon}$ is given by

$$
\mathbb{S}_{22}^{\varepsilon}\left(\mathrm{y}_{1}\right)=\left[\begin{array}{ccc}
\mathbb{B}_{11}^{\varepsilon}\left(\mathrm{y}_{1}\right) & \cdots & \mathbb{B}_{1 p}\left(\mathrm{y}_{1}\right) \\
\vdots & \ddots & \vdots \\
\mathbb{B}_{p 1}\left(\mathrm{y}_{1}\right) & \cdots & \mathbb{B}_{p p}^{\varepsilon}\left(\mathrm{y}_{1}\right)
\end{array}\right]
$$

where the blocks $\mathbb{B}_{i j}\left(\mathrm{y}_{1}\right)$ are defined by

$$
\begin{cases}\mathbb{B}_{i j}\left(\mathrm{y}_{1}\right)=\Delta t \mu_{i j} B_{i j} \operatorname{Diag}_{N \times N}\left(c_{i, \ell+\frac{1}{2}}^{n+1}\right) & \text { if } i \neq j, \\ \mathbb{B}_{i j}^{\varepsilon}\left(\mathrm{y}_{1}\right)=-\Delta t \operatorname{Diag}_{N \times N}\left(\kappa_{\ell}\right) & \text { if } i=j,\end{cases}
$$

where $\kappa_{\ell}=\sum_{k \neq i} \mu_{i k} B_{i k} c_{k, \ell+\frac{1}{2}}^{n+1}+\varepsilon^{2} \frac{m_{i}}{\Delta t}$, for any $0 \leq \ell \leq N-1$. System 22 with boundary conditions (20) is then solved by a Newton fixed-point method, with initial conditions satisfying the compatibility conditions (9).

\section{A PRIORI ESTIMATES FOR THE NUMERICAL SCHEME}

In this section, we prove that, for each species and all time, the scheme satisfies mass conservation, and the concentrations remain nonnegative if they are initially nonnegative.

Proposition 1. System (21) with boundary conditions (20) satisfies mass conservation for each species $\mathcal{A}_{i}, 1 \leq i \leq p$, i.e.

$$
\sum_{j=0}^{N} c_{i, j}^{n+1}=\sum_{j=0}^{N} c_{i, j}^{n}, \quad \forall n \in \mathbb{N} .
$$

Proof. For each species $\mathcal{A}_{i}, 1 \leq i \leq p$, we sum equations (17) over $0 \leq j \leq N$, we obtain

$$
\sum_{j=0}^{N} c_{i, j}^{n+1}=F_{i, N+\frac{1}{2}}^{n+1}-F_{i,-\frac{1}{2}}^{n+1}+\sum_{j=0}^{N} c_{i, j}^{n}, \quad \forall n \in \mathbb{N} .
$$

Boundary conditions 20 ensure the claimed result.

In order to prove nonnegativity of the solutions, we apply the method used in [1] and introduce the following auxiliary scheme

$$
\begin{aligned}
\left(-\Delta t \sum_{k \neq i} \mu_{i k} B_{i k}\left[c_{k, j+\frac{1}{2}}^{n+1}\right]^{+}-\varepsilon^{2} m_{i}\right) & F_{i, j+\frac{1}{2}}^{n+1}+\Delta t\left[c_{i, j+\frac{1}{2}}^{n+1}\right]^{+} \sum_{k \neq i} \mu_{i k} B_{i k} F_{k, j+\frac{1}{2}}^{n+1} \\
& =k_{B} T \lambda\left(c_{i, j+1}^{n+1}-c_{i, j}^{n+1}\right)+\varepsilon^{2} m_{i} \lambda R_{i, j+\frac{1}{2}}^{n}-\varepsilon^{2} m_{i} F_{i, j+\frac{1}{2}}^{n},
\end{aligned}
$$

with the notation $[c]^{+}=\max (c, 0)$ for any $c \in \mathbb{R}$. In the same way as for $(13)$, we can write these equations in a matrix form, introducing a modified matrix $\tilde{A}=\left([\tilde{A}]_{i k}\right)_{1 \leq i, k \leq p}$ defined by

$$
[\tilde{A}]_{i k}= \begin{cases}-\Delta t \mu_{i k} B_{i j}\left[c_{i}\right]^{+}, & \text {if } i \neq k, \\ \Delta t \sum_{\ell \neq i} \mu_{i \ell} B_{i \ell}\left[c_{\ell}\right]^{+}+\varepsilon^{2} m_{i}, & \text { if } i=k .\end{cases}
$$

Observe that this matrix depends on the concentrations of each species, and can therefore vary for any discrete time $t^{n}, n \geq 0$ and any point $x_{j}, j \in\left\{0, \frac{1}{2}, \cdots, N-\frac{1}{2}, N\right\}$. When needed, we denote this dependence in the following straightforward way $\tilde{A}_{j}^{n}=\left(\left[\tilde{A}_{j}^{n}\right]_{i k}\right)_{1 \leq i, k \leq p}$, for the same $j \in\left\{0, \frac{1}{2}, \cdots, N-\frac{1}{2}, N\right\}$

$$
\left[\tilde{A}_{j}^{n}\right]_{i k}= \begin{cases}-\Delta t \mu_{i k} B_{i j}\left[c_{i, j}^{n}\right]^{+}, & \text {if } i \neq k, \\ \Delta t \sum_{\ell \neq i} \mu_{i \ell} B_{i \ell}\left[c_{\ell, j}^{n}\right]^{+}+\varepsilon^{2} m_{i}, & \text { if } i=k .\end{cases}
$$


In this perspective, we can define, for any $n \geq 0$, the vectors of unknowns of all species

$$
\begin{aligned}
\mathcal{C}_{j}^{n} & =\left(c_{1, j}^{n}, \cdots, c_{p, j}^{n}\right)^{\top} \in \mathbb{R}^{p}, \quad 0 \leq j \leq N, \\
\mathcal{F}_{j+\frac{1}{2}}^{n} & =\left(F_{1, j+\frac{1}{2}}^{n}, \cdots, F_{p, j+\frac{1}{2}}^{n}\right)^{\top} \in \mathbb{R}^{p}, \quad 0 \leq j \leq N-1 .
\end{aligned}
$$

The continuity equations (17) can be written for any $n \in \mathbb{N}$ and any $1 \leq j \leq N-1$ as

$$
\frac{\mathcal{C}_{j}^{n+1}-\mathcal{C}_{j}^{n}}{\lambda}+\left(\mathcal{F}_{j+\frac{1}{2}}^{n+1}-\mathcal{F}_{j-\frac{1}{2}}^{n+1}\right)=0
$$

Equation (24) can also be written in a vectorial form for any $n \in \mathbb{N}$ and any $0 \leq j \leq N-1$

$$
-\tilde{A}_{j+\frac{1}{2}}^{n+1} \mathcal{F}_{j+\frac{1}{2}}^{n+1}=k_{B} T \lambda\left(\mathcal{C}_{j+1}^{n+1}-\mathcal{C}_{j}^{n+1}\right)+\varepsilon^{2} \mathcal{S}_{j+\frac{1}{2}}^{n},
$$

where the vectorial source term is defined by

$$
\mathcal{S}_{j+\frac{1}{2}}^{n}=\left(m_{1}\left(\lambda R_{1, j+\frac{1}{2}}^{n}-F_{1, j+\frac{1}{2}}^{n}\right), \cdots, m_{p}\left(\lambda R_{p, j+\frac{1}{2}}^{n}-F_{p, j+\frac{1}{2}}^{n}\right)\right)^{\top} \in \mathbb{R}^{p} .
$$

As we noted, $\tilde{A}$ is defined point-wise in time and space, and the proof of nonnegativity of the concentrations relies on some particular properties of this matrix $\tilde{A}$ (Lemmata 2 and 3 ), which are true for any time and any space point.

Lemma 2. The matrix $\tilde{A}$ defined by 25] is invertible for any $\varepsilon>0$, and its eigenvalues are positive.

Proof. We first observe that the matrix $\tilde{A}$ is diagonally dominant, since, for any $\varepsilon>0$ and $1 \leq i \leq p$,

$$
\left|[\tilde{A}]_{i i}\right|=\Delta t \sum_{\ell \neq i} \mu_{i \ell} B_{i \ell}\left[c_{\ell}\right]^{+}+\varepsilon^{2} m_{i}>\Delta t \sum_{\ell \neq i} \mu_{i \ell} B_{i \ell}\left[c_{\ell}\right]^{+}=\sum_{\ell \neq i}\left|[\tilde{A}]_{i \ell}\right|
$$

which ensures the invertibility. Furthermore, in order to prove the positivity of the eigenvalues, let us assume in a first step that $\left[c_{i}\right]^{+}>0$, for all $1 \leq i \leq p$. Then the matrix $\tilde{A}$ can be written under the following form $\tilde{A}=\Delta S \Delta^{-1}+D$, where $D$ and $\Delta$ are diagonal matrices defined by

$$
D=\varepsilon^{2} \operatorname{diag}\left(m_{1}, \cdots, m_{p}\right), \quad \Delta=\operatorname{diag}\left(\sqrt{\left[c_{1}\right]^{+}}, \cdots, \sqrt{\left[c_{p}\right]^{+}}\right),
$$

and matrix $S=\left(S_{i k}\right)_{1 \leq i, k \leq p}$ is defined by

$$
S_{i k}= \begin{cases}-\Delta t \mu_{i k} B_{i j} \sqrt{\left[c_{i}\right]^{+}\left[c_{k}\right]^{+}}, & \text {if } i \neq k, \\ \Delta t \sum_{\ell \neq i} \mu_{i \ell} B_{i \ell}\left[c_{\ell}\right]^{+}, & \text {if } i=k .\end{cases}
$$

This matrix $S$ is obviously symmetric and is also positive semidefinite, since for any $v \in \mathbb{R}^{p}$,

$$
v^{\top} S v=\Delta t \sum_{i, k=1}^{p} \mu_{i k} B_{i k}\left(\sqrt{\left[c_{i}\right]^{+}} v_{k}-\sqrt{\left[c_{k}\right]^{+}} v_{i}\right)^{2} \geq 0 .
$$

Thus, it follows that $\tilde{A}$ is similar to a symmetric matrix $S+D=\Delta^{-1} \tilde{A} \Delta$, which is positive definite for any $\varepsilon>0$, thus all its eigenvalues are positive.

Now, let us prove that the result also holds if only one of the $\left[c_{i}\right]^{+}$equals 0 . Without loss of generality, renumbering the species if necessary, we can assume that $\left[c_{p}\right]^{+}=0$, and $\left[c_{i}\right]^{+}>0$ for $1 \leq i \leq p-1$. Then $[\tilde{A}]_{p k}=0$, for any $1 \leq k \leq p-1$. In order to compute the eigenvalues of $\tilde{A}$, we can thus compute $\operatorname{det}\left(\tilde{A}-\sigma \mathbb{I}_{p}\right)$ using the cofactor expansion for this determinant with respect to the last row

$$
\operatorname{det}\left(\tilde{A}-\sigma \mathbb{I}_{p}\right)=\left(\Delta t \sum_{k \neq p} \mu_{p k} B_{p k}\left[c_{k}\right]^{+}+\varepsilon^{2} m_{p}-\sigma\right) \pi(\sigma),
$$

where $\pi(\sigma)$ is the characteristic polynomial of the $(p-1) \times(p-1)$ matrix coming from the first $p-1$ lines and columns of $\tilde{A}$. For this $(p-1) \times(p-1)$ matrix, we can apply the result of the previous 
step, since all $\left[c_{i}\right]^{+}>0$ for $1 \leq i \leq p-1$, which means that all its eigenvalues are positive, and thus all eigenvalues of $\tilde{A}$ are also positive. A backward induction reasoning allows to treat in a same way the case when more than one $\left[c_{i}\right]^{+}$is equal to zero, which concludes the proof.

We also need some properties on the elements of the inverse matrix $\tilde{A}^{-1}$, which are summed up in the following lemma.

Lemma 3. The coefficients of the matrix $\tilde{A}^{-1}$ are nonnegative. Moreover, its diagonal coefficients are positive, whereas all extra-diagonal terms of row $i, 1 \leq i \leq p$, contain a factor $\left[c_{i}\right]^{+}$.

Proof. The nonnegativity of matrix $\tilde{A}^{-1}$ comes from the $M$-matrices theory. Indeed, since all extradiagonal terms of $\tilde{A}$ are nonpositive, and since all eigenvalues of $\tilde{A}$ are positive (Lemma 2), matrix $\tilde{A}^{-1}$ is nonnegative [42, by Thm. 1, equivalence of $C_{9}$ and $F_{15}$ for matrices in $Z^{n, n}$ ], and its diagonal elements are positive [39, 7.10.12].

Consider an extra-diagonal term $\left[\tilde{A}^{-1}\right]_{i k}$ of $\tilde{A}^{-1}, 1 \leq i, k \leq p, i \neq k$. The determinant formula for inversion gives $\left[\tilde{A}^{-1}\right]_{i k}=\AA_{k i} / \operatorname{det}(\tilde{A}), \AA_{k i}$ being the cofactor of matrix $\tilde{A}$ associated with the $k$-th row and $i$-th column. To compute this cofactor, we have to compute the determinant of a matrix coming from $\tilde{A}$ without the $i$-th column of $\tilde{A}$, thus all elements of its $i$-th row contain $\left[c_{i}\right]^{+}$. Since obviously, $\operatorname{det}(\tilde{A})$ cannot be factorized by $\left[c_{i}\right]^{+}$, this concludes the proof.

Let us now state an a priori positivity result on the concentrations for our scheme (17) $-(19)$ with boundary conditions 20 . The existence of solutions to this scheme will be proved in the next section (Theorem 6). As mentioned earlier, the proof relies on the introduction of an auxiliary system:

$$
\left\{\begin{aligned}
& c_{i, j}^{n+1}+\lambda\left(F_{i, j+\frac{1}{2}}^{n+1}-F_{i, j-\frac{1}{2}}^{n+1}\right)=c_{i, j}^{n}, \\
&\left(-\Delta t \sum_{k \neq i} \mu_{i k} B_{i k}\left[c_{k, j+\frac{1}{2}}^{n+1}\right]^{+}-\varepsilon^{2} m_{i}\right) F_{i, j+\frac{1}{2}}^{n+1}+\Delta t\left[c_{i, j+\frac{1}{2}}^{n+1}\right]^{+} \sum_{k \neq i} \mu_{i k} B_{i k} F_{k, j+\frac{1}{2}}^{n+1} \\
&=k_{B} T \lambda\left(c_{i, j+1}^{n+1}-c_{i, j}^{n+1}\right)+\varepsilon^{2} m_{i} \lambda R_{i, j+\frac{1}{2}}^{n}-\varepsilon^{2} m_{i} F_{i, j+\frac{1}{2}}^{n}
\end{aligned}\right.
$$

In order to precisely state the boundedness assumption needed on the source terms in Proposition 4, let us define the quantity

$$
M_{\varepsilon}=\varepsilon^{2} \max _{1 \leq i \leq p} \max _{n \geq 0} \max _{0 \leq j \leq N-1}\left|\mathcal{S}_{i, j+\frac{1}{2}}^{n}\right| .
$$

We need the source terms to be uniformly bounded, which is guaranteed by

$$
\lim _{\varepsilon \rightarrow 0} M_{\varepsilon}=0 \text {. }
$$

Proposition 4. Assume that for any $1 \leq i \leq p$ and any $n \geq 0$, there exist solutions $\left(c_{i, j}^{n}\right)_{0 \leq j \leq N}$, $\left(F_{i, j+\frac{1}{2}}^{n}\right)_{0 \leq j \leq N-1}$ of the auxiliary system (28) with boundary conditions (20). Assume also that (30) holds. If, for any $1 \leq i \leq p, 0 \leq j \leq N, c_{i, j}^{i n} \geq 0$, then for any $n \in \mathbb{N}$ and $\varepsilon$ small enough, $c_{i, j}^{n} \geq 0$. Moreover, $\left(c_{i, j}^{n}\right)_{0 \leq j \leq N},\left(F_{i, j+\frac{1}{2}}^{n}\right)_{0 \leq j \leq N-1}$ are also solutions of the initial system 21] with boundary conditions 20.

Proof. Let us prove this result by induction. The base case is obviously true by assumption. Assume that $c_{i, j}^{n} \geq 0$, for any $1 \leq i \leq p, 0 \leq j \leq N$. Since $\tilde{A}_{j+\frac{1}{2}}^{n+1}$ is invertible for any $0 \leq j \leq N-1$ and $n \in \mathbb{N}$, we can rewrite (27) as

$$
\mathcal{F}_{j+\frac{1}{2}}^{n+1}=-\left(\tilde{A}_{j+\frac{1}{2}}^{n+1}\right)^{-1}\left(k_{B} T \lambda\left(\mathcal{C}_{j+1}^{n+1}-\mathcal{C}_{j}^{n+1}\right)+\varepsilon^{2} \mathcal{S}_{j+\frac{1}{2}}^{n}\right) .
$$

Injecting this expression in the continuity equations (26), we obtain multi-species diffusion equations for $1 \leq j \leq N-1$, 


$$
\begin{aligned}
\frac{\mathcal{C}_{j}^{n+1}-\mathcal{C}_{j}^{n}}{\lambda}=\left(\tilde{A}_{j+\frac{1}{2}}^{n+1}\right)^{-1}\left(k_{B} T \lambda\left(\mathcal{C}_{j+1}^{n+1}-\mathcal{C}_{j}^{n+1}\right)\right. & \left.+\varepsilon^{2} \mathcal{S}_{j+\frac{1}{2}}^{n}\right) \\
& -\left(\tilde{A}_{j-\frac{1}{2}}^{n+1}\right)^{-1}\left(k_{B} T \lambda\left(\mathcal{C}_{j}^{n+1}-\mathcal{C}_{j-1}^{n+1}\right)+\varepsilon^{2} \mathcal{S}_{j-\frac{1}{2}}^{n}\right),
\end{aligned}
$$

and using boundary conditions (20), we obtain the two additional equations

$$
\begin{aligned}
& \frac{\mathcal{C}_{0}^{n+1}-\mathcal{C}_{0}^{n}}{\lambda}=\left(\tilde{A}_{\frac{1}{2}}^{n+1}\right)^{-1}\left(k_{B} T \lambda\left(\mathcal{C}_{1}^{n+1}-\mathcal{C}_{0}^{n+1}\right)+\varepsilon^{2} \mathcal{S}_{\frac{1}{2}}^{n}\right), \\
& \frac{\mathcal{C}_{N}^{n+1}-\mathcal{C}_{N}^{n}}{\lambda}=-\left(\tilde{A}_{N-\frac{1}{2}}^{n+1}\right)^{-1}\left(k_{B} T \lambda\left(\mathcal{C}_{N}^{n+1}-\mathcal{C}_{N-1}^{n+1}\right)+\varepsilon^{2} \mathcal{S}_{N-\frac{1}{2}}^{n}\right) .
\end{aligned}
$$

It is then possible to apply the same approach as in [1] to these multi-species diffusion equations. Let us denote by $\langle u, v\rangle_{p}=\sum_{i=1}^{p} u_{i} v_{i}$, for $u, v \in \mathbb{R}^{p}$ the usual scalar product in $\mathbb{R}^{p}$, and define the vectorial negative part of the concentrations $\left[\mathcal{C}_{j}^{n}\right]^{-}=\left(\left[c_{1, j}^{n}\right]^{-}, \cdots,\left[c_{p, j}^{n}\right]^{-}\right)^{\top} \in \mathbb{R}^{p}, \quad 0 \leq j \leq N$, with the notation $[c]^{-}=\max (-c, 0)$ for any $c \in \mathbb{R}$. Now, we sum equations (31) for $1 \leq j \leq N-1$ and both equations in 32 , we compute the scalar product in $\mathbb{R}^{p}$ with $\left[\mathcal{C}_{j}^{n+1}\right]^{-}$, and perform a discrete integration by parts to obtain

$$
\begin{aligned}
\sum_{j=0}^{N}\left\langle\frac{\mathcal{C}_{j}^{n+1}-\mathcal{C}_{j}^{n}}{\lambda},\left[\mathcal{C}_{j}^{n+1}\right]^{-}\right\rangle_{p}+k_{B} T \lambda \sum_{j=0}^{N}\left\langle\left(\tilde{A}_{j+\frac{1}{2}}^{n+1}\right)^{-1}\left(\mathcal{C}_{j+1}^{n+1}-\mathcal{C}_{j}^{n+1}\right),\left(\left[\mathcal{C}_{j+1}^{n+1}\right]^{-}-\left[\mathcal{C}_{j}^{n+1}\right]^{-}\right)\right\rangle_{p} \\
+\varepsilon^{2} \sum_{j=0}^{N}\left\langle\left(\tilde{A}_{j+\frac{1}{2}}^{n+1}\right)^{-1} \mathcal{S}_{j+\frac{1}{2}}^{n},\left(\left[\mathcal{C}_{j+1}^{n+1}\right]^{-}-\left[\mathcal{C}_{j}^{n+1}\right]^{-}\right)\right\rangle_{p}=0 .
\end{aligned}
$$

Let us momentarily fix $0 \leq j \leq N$ and consider the terms involving the product with the matrix $\left(\tilde{A}_{j+\frac{1}{2}}^{n+1}\right)^{-1}$. Expanding the product, there are terms involving the extra-diagonal terms of the matrix, which are written, for any $1 \leq i, k \leq p, k \neq i$

$$
T_{i k}=\left[\left(\tilde{A}_{j+\frac{1}{2}}^{n+1}\right)^{-1}\right]_{i k}\left(k_{B} T \lambda\left(c_{k, j+1}^{n+1}-c_{k, j}^{n+1}\right)+\varepsilon^{2} \mathcal{S}_{k, j+\frac{1}{2}}^{n}\right)\left(\left[c_{i, j+1}^{n+1}\right]^{-}-\left[c_{i, j}^{n+1}\right]^{-}\right),
$$

and terms involving the diagonal terms of $\left(\tilde{A}_{j+\frac{1}{2}}^{n+1}\right)^{-1}$, which are written for any $1 \leq i \leq p$

$$
T_{i i}=\left[\left(\tilde{A}_{j+\frac{1}{2}}^{n+1}\right)^{-1}\right]_{i i}\left(k_{B} T \lambda\left(c_{i, j+1}^{n+1}-c_{i, j}^{n+1}\right)+\varepsilon^{2} \mathcal{S}_{i, j+\frac{1}{2}}^{n}\right)\left(\left[c_{i, j+1}^{n+1}\right]^{-}-\left[c_{i, j}^{n+1}\right]^{-}\right) .
$$

Thanks to Lemma 3, we know that all terms $T_{i k}$ contain a factor $\left[c_{i, j+\frac{1}{2}}^{n+1}\right]^{+}$. Using the definition (18) of $c_{i, j+\frac{1}{2}}$, we deduce that all $T_{i k}$ contain a factor $\min \left\{\left[c_{i, j}^{n+1}\right]^{+},\left[c_{i, j+1}^{n+1}\right]^{+}\right\}\left(\left[c_{i, j+1}^{n+1}\right]^{-}-\left[c_{i, j}^{n+1}\right]^{-}\right)$, which always equals 0 . This ensures that $T_{i k}=0$.

As far as the diagonal terms $T_{i i}$ are concerned, we know from Lemma 3 that $\left[\left(\tilde{A}_{j+\frac{1}{2}}^{n+1}\right)^{-1}\right]_{i i} \geq 0$. Moreover, observe that $\left(c_{i, j+1}^{n+1}-c_{i, j}^{n+1}\right)\left(\left[c_{i, j+1}^{n+1}\right]^{-}-\left[c_{i, j}^{n+1}\right]^{-}\right) \leq 0$, for any values of the concentrations. The term involving $\mathcal{S}_{i, j+\frac{1}{2}}^{n}$ has an undefined sign, but the assumption of $\mathcal{S}_{i, j+\frac{1}{2}}^{n}$ ensures that it remains controlled, for $\varepsilon$ small enough, by the first term. Thus, if $\left(c_{i, j+1}^{n+1}-c_{i, j}^{n+1}\right)\left(\left[c_{i, j+1}^{n+1}\right]^{-}-\left[c_{i, j}^{n+1}\right]^{-}\right)<$ 0 , for $\varepsilon$ small enough, we can ensure that $T_{i i}$ remains negative. Moreover, it is obvious that if $\left(c_{i, j+1}^{n+1}-c_{i, j}^{n+1}\right)\left(\left[c_{i, j+1}^{n+1}\right]^{-}-\left[c_{i, j}^{n+1}\right]^{-}\right)=0$, then $\left[c_{i, j+1}^{n+1}\right]^{-}-\left[c_{i, j}^{n+1}\right]^{-}=0$, which ensures that $T_{i i}=0$. Finally, it follows that

$$
\sum_{j=0}^{N}\left\langle\mathcal{C}_{j}^{n+1}-\mathcal{C}_{j}^{n},\left[\mathcal{C}_{j}^{n+1}\right]^{-}\right\rangle_{p} \geq 0
$$


Using that $\mathcal{C}_{j}^{n+1}=\left[\mathcal{C}_{j}^{n+1}\right]^{+}-\left[\mathcal{C}_{j}^{n+1}\right]^{-}$, and that $\left\langle\left[\mathcal{C}_{j}^{n+1}\right]^{+},\left[\mathcal{C}_{j}^{n+1}\right]^{-}\right\rangle_{p}=0$, we deduce that

$$
-\sum_{j=0}^{N}\left\langle\left[\mathcal{C}_{j}^{n+1}\right]^{-},\left[\mathcal{C}_{j}^{n+1}\right]^{-}\right\rangle_{p} \geq \sum_{j=0}^{N}\left\langle\mathcal{C}_{j}^{n},\left[\mathcal{C}_{j}^{n+1}\right]^{-}\right\rangle_{p}
$$

Since $c_{i, j}^{n} \geq 0$ for any $1 \leq i \leq p, 0 \leq j \leq N$ by induction hypothesis, this implies that $\sum_{j=0}^{N}\left|\left[\mathcal{C}_{j}^{n+1}\right]^{-}\right|_{p}^{2} \leq$ 0 , where $|\cdot|_{p}$ is the norm associated to the scalar product $\langle\cdot, \cdot\rangle_{p}$. Therefore, $\left[c_{i, j}^{n+1}\right]^{-}=0$ for any $1 \leq i \leq p, 0 \leq j \leq N$, which means that $c_{i, j}^{n+1} \geq 0$, and concludes the proof by induction.

Last, we note that a solution $\left(c_{i, j}^{n}\right)_{0 \leq j \leq N},\left(F_{i, j+\frac{1}{2}}^{n}\right)_{0 \leq j \leq N-1}$ of the auxiliary system 28) is also solution of (21) because of the nonnegativity of $\left(c_{i, j}^{n}\right)_{0 \leq j \leq N}$, which implies that $\left[c_{i, j}^{n+1}\right]^{+}=c_{i, j}^{n+1}$.

Remark 5. Assumption (30) stating that the source terms $\mathcal{S}_{i, j+\frac{1}{2}}^{n}$ is bounded for any $1 \leq i \leq p$, $0 \leq j \leq N-1, n \in \mathbb{N}$ uniformly with respect to $\varepsilon$, is satisfied if the nonlinear terms (discretization of $\partial_{x}\left(c u^{2}\right)$ ) and the fluxes remain uniformly bounded. This assumption will be numerically checked a posteriori in Section 5 for the numerical tests.

\section{Existence of a solution to the numerical scheme}

It remains to prove the existence of a solution to our scheme. To this end, we use a matrix form similar to 22 of a new auxiliary system, inspired from (28). Let

$$
\tilde{\mathrm{y}}=\left(\tilde{\mathrm{y}}_{1}, \mathrm{y}_{2}\right)^{\top}=\left(\left[c_{1,0}\right]^{+}, \cdots,\left[c_{1, N}\right]^{+}, \cdots,\left[c_{p, 0}\right]^{+}, \cdots,\left[c_{p, N}\right]^{+}, \mathrm{y}_{2}\right)^{\top} \in \mathbb{R}^{p(2 N+1)} .
$$

Then we prove the existence of a solution to the following new auxiliary system

$$
\left\{\begin{array}{r}
{\left[c_{i, j}^{n+1}\right]^{+}+\lambda\left(F_{i, j+\frac{1}{2}}^{n+1}-F_{i, j-\frac{1}{2}}^{n+1}\right)=c_{i, j}^{n}} \\
\left(-\Delta t \sum_{k \neq i} \mu_{i k} B_{i k}\left[c_{k, j+\frac{1}{2}}^{n+1}\right]^{+}-\varepsilon^{2} m_{i}\right) F_{i, j+\frac{1}{2}}^{n+1}+\Delta t\left[c_{i, j+\frac{1}{2}}^{n+1}\right]^{+} \sum_{k \neq i} \mu_{i k} B_{i k} F_{k, j+\frac{1}{2}}^{n+1} \\
\quad=k_{B} T \lambda\left(\left[c_{i, j+1}^{n+1}\right]^{+}-\left[c_{i, j}^{n+1}\right]^{+}\right)+\varepsilon^{2} m_{i} \lambda R_{i, j+\frac{1}{2}}^{n}-\varepsilon^{2} m_{i} F_{i, j+\frac{1}{2}}^{n}
\end{array}\right.
$$

This system (33) can be written as

$$
\tilde{\mathbb{S}}^{\varepsilon}\left(\tilde{\mathrm{y}}_{1}^{n+1}\right) \tilde{\mathrm{y}}^{n+1}=\mathrm{b}^{n}, \text { where } \tilde{\mathbb{S}}^{\varepsilon}\left(\tilde{\mathrm{y}}_{1}\right)=\left[\begin{array}{cc}
\mathbb{S}_{11} & \mathbb{S}_{12} \\
\mathbb{S}_{21} & \tilde{\mathbb{S}}_{22}^{\varepsilon}\left(\tilde{\mathrm{y}}_{1}\right)
\end{array}\right],
$$

and $\tilde{\mathbb{S}}_{22}^{\varepsilon}$ is given by

$$
\tilde{\mathbb{S}}_{22}^{\varepsilon}\left(\tilde{\mathrm{y}}_{1}\right)=\left[\begin{array}{ccc}
\tilde{\mathbb{B}}_{11}^{\varepsilon}\left(\tilde{\mathrm{y}}_{1}\right) & \cdots & \tilde{\mathbb{B}}_{1 p}\left(\tilde{\mathrm{y}}_{1}\right) \\
\vdots & \ddots & \vdots \\
\tilde{\mathbb{B}}_{p 1}\left(\tilde{\mathrm{y}}_{1}\right) & \cdots & \tilde{\mathbb{B}}_{p p}^{\varepsilon}\left(\tilde{\mathrm{y}}_{1}\right)
\end{array}\right]
$$

where the blocks $\tilde{\mathbb{B}}_{i j}\left(\tilde{y}_{1}\right)$ are defined by

$$
\left\{\begin{array}{l}
\tilde{\mathbb{B}}_{i j}\left(\tilde{\mathrm{y}}_{1}\right)=\Delta t \mu_{i j} B_{i j} \operatorname{Diag}_{N \times N}\left(\left[c_{i, \ell-\frac{1}{2}}^{n+1}\right]^{+}\right) \text {if } i \neq j, \\
\tilde{\mathbb{B}}_{i i}^{\varepsilon}\left(\tilde{\mathrm{y}}_{1}\right)=-\Delta t \operatorname{Diag}_{N \times N}\left(\bar{\kappa}_{\ell}\right),
\end{array}\right.
$$

where $\bar{\kappa}_{\ell}=\sum_{k \neq i} \mu_{i k} B_{i k}\left[c_{k, \ell-\frac{1}{2}}^{n+1}\right]^{+}+\varepsilon^{2} \frac{m_{i}}{\Delta t}$, for $1 \leq \ell \leq N$.

Theorem 6. For any $\varepsilon>0$, there exists a solution $\left(c_{i, j}^{n}\right)_{0 \leq j \leq N},\left(F_{i, j+\frac{1}{2}}^{n}\right)_{0 \leq j \leq N-1}$ of the system (33) with boundary conditions (20), which also solves (28). Moreover, thanks to Proposition 4, for $\varepsilon>0$ small enough, it is also a solution of system (21) with boundary conditions (20). 
Proof. First, let us prove that $\tilde{\mathbb{S}}^{\varepsilon}\left(\tilde{\mathrm{y}}_{1}\right)$ is invertible for any $\tilde{\mathrm{y}}_{1}$ and any $\varepsilon>0$. Since $\mathbb{S}_{11}=\mathbb{I}_{p(N+1)}$, it is obviously invertible of determinant 1, and block determinant computation using the Schur complement leads to

$$
\operatorname{det}\left(\tilde{\mathbb{S}}^{\varepsilon}\left(\tilde{y}_{1}\right)\right)=\operatorname{det}\left(\tilde{\mathbb{S}}_{22}^{\varepsilon}\left(\tilde{y}_{1}\right)-\mathbb{S}_{21} \mathbb{S}_{12}\right)
$$

Let us denote $\tilde{\mathbb{P}}^{\varepsilon}\left(\tilde{\mathrm{y}}_{1}\right)=\tilde{\mathbb{S}}_{22}^{\varepsilon}\left(\tilde{\mathrm{y}}_{1}\right)-\mathbb{S}_{21} \mathbb{S}_{12}$. Obviously, proving that $\tilde{\mathbb{P}}^{\varepsilon}\left(\tilde{\mathrm{y}}_{1}\right)$ is invertible is enough to prove that $\tilde{\mathbb{S}}^{\varepsilon}\left(\tilde{\mathrm{y}}_{1}\right)$ also is. We compute

$$
\mathbb{S}_{21} \mathbb{S}_{12}=k_{B} T \lambda^{2}\left(\begin{array}{ccccc}
2 & -1 & & \cdots & 0 \\
-1 & 2 & \ddots & & \vdots \\
0 & \ddots & \ddots & \ddots & \vdots \\
\vdots & & \ddots & \ddots & -1 \\
0 & \cdots & \cdots & -1 & 2
\end{array}\right) \in \mathbb{R}^{p N \times p N} .
$$

It follows that the diagonal terms of $\tilde{\mathbb{P}}^{\varepsilon}\left(\tilde{y}_{1}\right)$ are negative, whereas its extra-diagonal terms are nonnegative. Moreover, the transpose of matrix $\tilde{\mathbb{P}}^{\varepsilon}\left(\tilde{y}_{1}\right)$ is also diagonally dominant, since for any $\varepsilon>0, n \geq 0$ and any $1 \leq q \leq p N$

$$
\begin{aligned}
\left|\left[\tilde{\mathbb{P}}^{\varepsilon}\left(\tilde{\mathrm{y}}_{1}\right)^{\top}\right]_{q q}\right|=\sum_{k \neq i} \Delta t \mu_{i k} B_{i k}\left[c_{k, \ell-\frac{1}{2}}^{n+1}\right]^{+}+m_{i} \varepsilon^{2}+2 k_{B} T \lambda^{2} \\
\quad>\sum_{k \neq i} \Delta t \mu_{i k} B_{i k}\left[c_{k, \ell-\frac{1}{2}}^{n+1}\right]^{+}+2 k_{B} T \lambda^{2} \geq \sum_{r \neq q}\left|\left[\tilde{\mathbb{P}}^{\varepsilon}\left(\tilde{\mathrm{y}}_{1}\right)^{\top}\right]_{q r}\right|
\end{aligned}
$$

where $i=\left\lfloor\frac{q-1}{N}\right\rfloor+1$, and $\ell=q-N\left\lfloor\frac{q-1}{N}\right\rfloor$. This proves the invertibility of $\tilde{\mathbb{P}}^{\varepsilon}\left(\tilde{\mathrm{y}}_{1}\right)$, and thus the one of $\tilde{\mathbb{S}}^{\varepsilon}\left(\tilde{y}_{1}\right)$. Since $\tilde{\mathbb{S}}^{\varepsilon}\left(\tilde{y}_{1}\right)$ is invertible for any $\tilde{y}_{1}$ and any $\varepsilon>0$, the new auxiliary system (33) can be written under the form

$$
\tilde{y}^{n+1}=\left(\tilde{\mathbb{S}}^{\varepsilon}\left(\tilde{y}_{1}^{n+1}\right)\right)^{-1} \mathrm{~b}^{n}
$$

where $\mathrm{b}^{n}=\left(\mathrm{b}_{1}^{n}, \mathrm{~b}_{2}^{n}\right)^{\top}$ is defined by (23). The existence of a solution to this system is then proved using Schaefer's fixed-point theorem. To apply this fixed-point theorem, we compute, again thanks to the Schur complement,

$$
\left(\tilde{\mathbb{S}}^{\varepsilon}\left(\tilde{\mathrm{y}}_{1}^{n+1}\right)\right)^{-1}=\left[\begin{array}{cc}
\mathbb{I}_{p(N+1)}+\mathbb{S}_{12}\left(\tilde{\mathbb{P}}^{\varepsilon}\left(\tilde{\mathrm{y}}_{1}^{n+1}\right)\right)^{-1} \mathbb{S}_{21} & -\mathbb{S}_{12}\left(\tilde{\mathbb{P}}^{\varepsilon}\left(\tilde{\mathrm{y}}_{1}^{n+1}\right)\right)^{-1} \\
-\left(\tilde{\mathbb{P}}^{\varepsilon}\left(\tilde{\mathrm{y}}_{1}^{n+1}\right)\right)^{-1} \mathbb{S}_{21} & \left(\tilde{\mathbb{P}}^{\varepsilon}\left(\tilde{\mathrm{y}}_{1}^{n+1}\right)\right)^{-1}
\end{array}\right]
$$

which means that we can define two functions $f$ ang $g$ so that the system can also be written as

$$
\left\{\begin{array}{l}
\tilde{\mathrm{y}}_{1}^{n+1}=f\left(\tilde{\mathrm{y}}_{1}^{n+1}\right)=\mathrm{b}_{1}^{n}+\mathbb{S}_{12}\left(\tilde{\mathbb{P}}^{\varepsilon}\left(\tilde{\mathrm{y}}_{1}^{n+1}\right)\right)^{-1} \mathbb{S}_{21} \mathrm{~b}_{1}^{n}-\mathbb{S}_{12}\left(\tilde{\mathbb{P}}^{\varepsilon}\left(\tilde{\mathrm{y}}_{1}^{n+1}\right)\right)^{-1} \mathrm{~b}_{2}^{n}, \\
\mathrm{y}_{2}^{n+1}=g\left(\tilde{\mathrm{y}}_{1}^{n+1}\right)=-\left(\tilde{\mathbb{P}}^{\varepsilon}\left(\tilde{\mathrm{y}}_{1}^{n+1}\right)\right)^{-1} \mathbb{S}_{21} \mathrm{~b}_{1}^{n}+\left(\tilde{\mathbb{P}}^{\varepsilon}\left(\tilde{\mathrm{y}}_{1}^{n+1}\right)\right)^{-1} \mathrm{~b}_{2}^{n}
\end{array}\right.
$$

The proof principle is the following: we prove that there exists a solution to the first equation $\tilde{\mathrm{y}}_{1}^{n+1}=f\left(\tilde{\mathrm{y}}_{1}^{n+1}\right)$, then the existence of $\mathrm{y}_{2}$ immediately follows, in view of the second equation.

First, observe that the application $f$ maps $\left(\mathbb{R}^{+}\right)^{p(N+1)}$ to $\left(\mathbb{R}^{+}\right)^{p(N+1)}$, thanks to Proposition 4 , Moreover, it is continuous, since $\left(\tilde{\mathbb{P}}^{\varepsilon}\left(\tilde{\mathrm{y}}_{1}^{n+1}\right)\right)^{-1}$ is the inverse of an affine function. Since we work in a finite-dimensional setting, $f$ is also compact. Endow $\mathbb{R}^{p(N+1)}$ with the discrete $L^{1}$-norm $\|v\|_{1}=$ $\sum_{k=1}^{p(N+1)}\left|v_{k}\right|$, for any $v \in \mathbb{R}^{p(N+1)}$. The set

$$
E=\left\{\tilde{\mathrm{y}}_{1} \in\left(\mathbb{R}^{+}\right)^{p(N+1)} \mid \exists \xi \in[0,1] \text { such that } \tilde{\mathrm{y}}_{1}=\xi f\left(\tilde{\mathrm{y}}_{1}\right)\right\}
$$


is bounded. Indeed, let $\tilde{\mathrm{y}}_{1} \in E$, and $\xi \in[0,1]$ such that $\tilde{\mathrm{y}}_{1}=\xi f\left(\tilde{\mathrm{y}}_{1}\right)$. Define $\mathrm{y}_{2}=\xi g\left(\tilde{\mathrm{y}}_{1}\right)$, and $\tilde{y}=\left(\tilde{y}_{1}, y_{2}\right)^{\top}$. Then $\tilde{y}$ is solution of the equation

$$
\tilde{\mathbb{S}}^{\varepsilon}\left(\tilde{\mathrm{y}}_{1}\right) \tilde{\mathrm{y}}=\xi \mathrm{b} .
$$

Now, observe that as for recovering mass conservation (Proposition 1), if we sum the first equations of (33) over $i$ and $j$, we obtain

$$
\sum_{\substack{1 \leq i \leq p \\ 0 \leq j \leq N}}\left[c_{i, j}^{n+1}\right]^{+}=\sum_{\substack{1 \leq i \leq p \\ 0 \leq j \leq N}} c_{i, j}^{n},
$$

which means that $\left\|\tilde{y}_{1}^{n+1}\right\|_{1}=\left\|b_{1}^{n}\right\|_{1}$ by definition of $b_{1}$. With the same reasoning, a solution $\tilde{y}_{1}$ of (34) satisfies

$$
\left\|\tilde{y}_{1}^{n+1}\right\|_{1}=\xi\left\|b_{1}^{n}\right\|_{1} \leq\left\|b_{1}^{n}\right\|_{1}, \quad \forall \xi \in[0,1] .
$$

This proves that the set $E$ is bounded. Therefore, Schaefer's fixed point theorem [19, Thm. 4, p. 509] ensures that $f$ has a fixed point $\tilde{y}_{1}^{n+1}$, and $\tilde{y}^{n+1}=\left(\tilde{y}_{1}^{n+1}, \mathrm{y}_{2}^{n+1}\right)^{\top}$ is thus a solution of the new auxiliary system (33). By construction, since we added positive parts on every concentration in this new auxiliary system, all components of $\tilde{y}_{1}$ are nonnegative, which means that it is also a solution to the auxiliary system (28). Finally, Proposition 4 ensures that it is also a solution to (21).

\section{NUMERICAL EXPERIMENTS}

In this section, we illustrate the good behavior of the scheme on some numerical examples.

5.1. Parameters of the scheme, validation. The numerical values of the different constants involved in the equations are the following.

- The temperature $T$ is given by $T=300 \mathrm{~K}$, and $k_{B}=\mathcal{N}_{A} R$, where $R=8.314 \mathrm{~J} \cdot \mathrm{mol}^{-1} \cdot \mathrm{K}^{-1}$ and $\mathcal{N}_{A}$ is the Avogadro constant. The equations for the fluxes are actually multiplied by $\mathcal{N}_{A}$, so that we can use the molar masses of each species.

- Species 1, 2 and 3 have respective molar masses $M_{1}=2 \mathrm{~g} \cdot \mathrm{mol}^{-1}, M_{2}=28 \mathrm{~g} \cdot \mathrm{mol}^{-1}$ and $M_{3}=44 \mathrm{~g} \cdot \mathrm{mol}^{-1}$. These values respectively correspond to hydrogen, nitrogen and carbon dioxide.

- The cross sections are computed from the binary diffusion coefficients $D_{i j}$ through the formula $\tilde{B}_{i j}=\frac{\left(m_{i}+m_{j}\right) k_{B} T}{4 \pi m_{i} m_{j} D_{i j}}$, with $D_{12}=0.833 \mathrm{~cm}^{2} \cdot \mathrm{s}^{-1}, D_{13}=0.68 \mathrm{~cm}^{2} \cdot \mathrm{s}^{-1}$ and $D_{23}=$ $0.168 \mathrm{~cm}^{2} \cdot \mathrm{s}^{-1}$. In the computations, we chose to rescale them by a factor $10^{5}$ (which corresponds to accelerating time) to the values $B_{12}=0.802 \times 10^{3} \mathrm{~s}^{-1}, B_{13}=0.958 \times 10^{3} \mathrm{~s}^{-1}$, $B_{23}=0.433 \times 10^{3} \mathrm{~s}^{-1}$.

- The spatial domain $\Omega$ is chosen as $\Omega=[-1,1]$, with space step $\Delta x=10^{-2}$ and $\Delta t=\Delta x^{2}=$ $10^{-4}$ (obtained after performing the scaling). Observe that the discretization parameters are constant for all simulations we performed, and in particular that they are chosen a priori, not depending on $\varepsilon$.

Remark 7. The kinetic model (7) we use actually holds only for monoatomic gases. However, the macroscopic diffusion model (3) is valid for more general gases, including polyatomic ones. Since we aim to mimic Duncan and Toor's experiment [18, and in particular to observe uphill diffusion, we chose to simulate the behavior of hydrogen, nitrogen and carbon dioxide, which are not monoatomic gases.

First, we numerically check that constant solutions are preserved by the discretization, since constant states are exact solutions of our scheme. Indeed, for constant initial concentrations and zero initial fluxes, the scheme preserves the initial state and the mixture does not move from its equilibrium. 

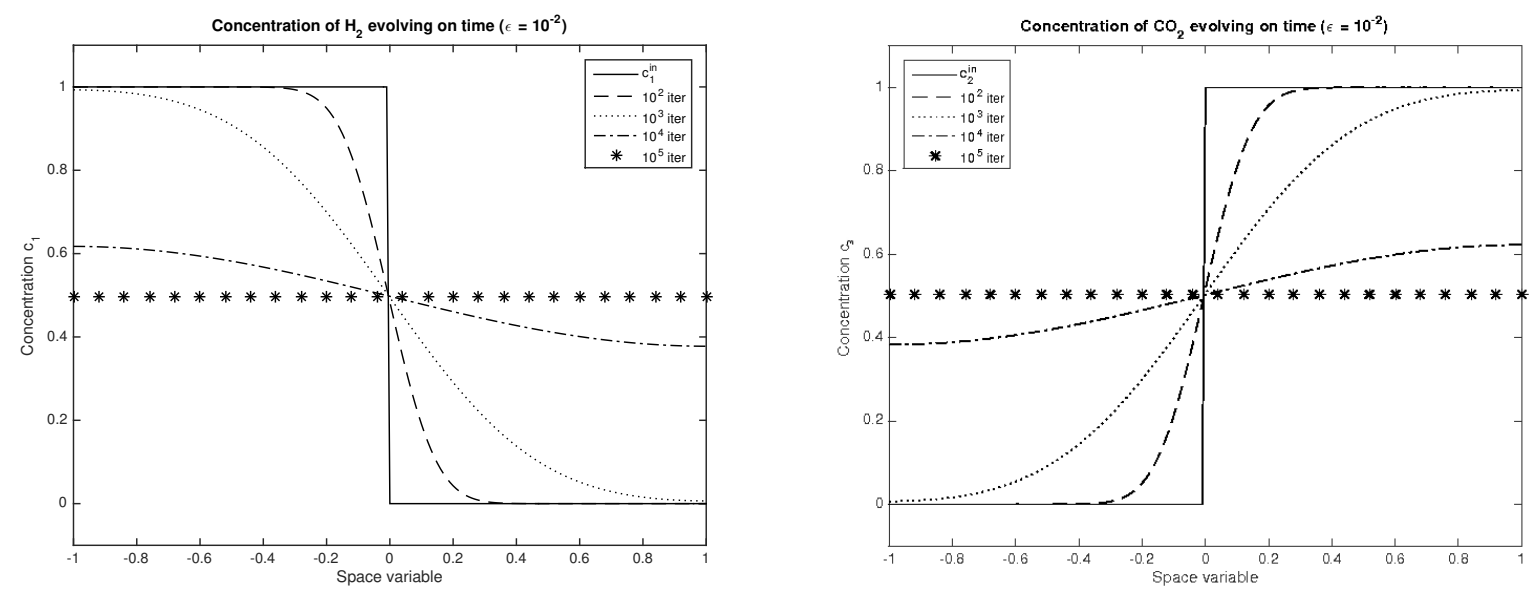

Figure 1. Evolution of the concentrations of species 1 and 2 in time in the twospecies test case

5.2. Two-species diffusion. The first test case we consider consists in a two-species mixture (species 1 and 3). In this case, the limit model (3) reduces to a simple heat equation on each species, since the cross-diffusion effects cancel due to the symmetry of the diffusion coefficients. We consider the following initial conditions

$$
c_{1}^{\text {in }}(x)=\mathbb{1}_{[-1,0]}, \quad c_{3}^{\text {in }}(x)=\mathbb{1}_{[0,1]}, \quad F_{1}^{\text {in }}(x)=F_{3}^{\text {in }}(x)=0 .
$$

For $\varepsilon=10^{-2}$, we plot on Figure 1 the concentrations of each species for various times $(t=0$, $t=10^{-2}, t=10^{-1}, t=1$ and $t=10$, since $\left.\Delta t=10^{-4}\right)$. Both species have the expected behavior and diffuse until reaching the equilibrium.

5.2.1. Discussion about the closure relation for the Maxwell-Stefan equations. As mentioned earlier, Maxwell-Stefan equations (3) need an additional closure relation to be solved, and a possible one is the equimolar diffusion setting, in which $c$ is identically equal to 1 . The initial conditions in our scheme are chosen in (9) in order to be compatible with this condition. However, the evolution in space and time of the total concentration is not imposed. In order to be consistent with the closure relation in the Maxwell-Stefan equations, we a posteriori check, at least numerically, that the total concentration remains close to 1 for any time and space. This is shown in Figure 2 .

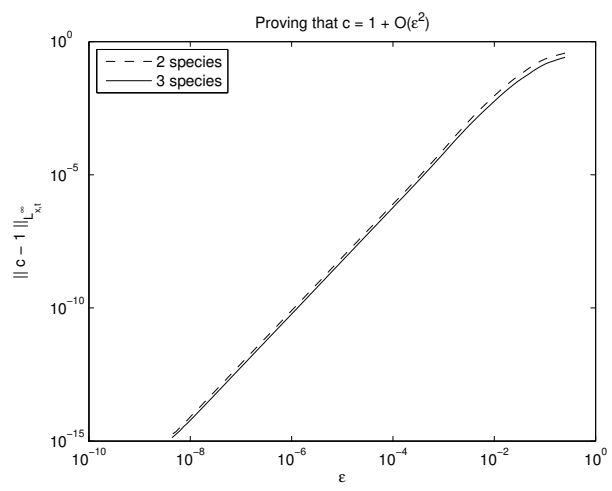

FIgURE 2. $L^{\infty}$-norm of $c-1$ with respect to $\varepsilon$

We observe that $c=1+O\left(\varepsilon^{2}\right)$, and in particular, for small values of $\varepsilon$, the closure relation is completely consistent with Boltzmann equations for mixtures. 
5.2.2. Numerical verification of Assumption (30) on the source terms. As stated in Remark 5, the proof of Proposition 4 relies on the assumption that the source terms $\mathcal{S}_{i, j+\frac{1}{2}}^{n}$ are bounded for any $1 \leq i \leq p, 0 \leq j \leq N-1, n \in \mathbb{N}$, uniformly with respect to $\varepsilon$. This assumption is a posteriori numerically checked in each test case. More precisely, if we recall the quantity $M_{\varepsilon}$ defined by $(29)$, we need $M_{\varepsilon}$ to become smaller when $\varepsilon$ vanishes, which is the case, as shown on Figure 3 .

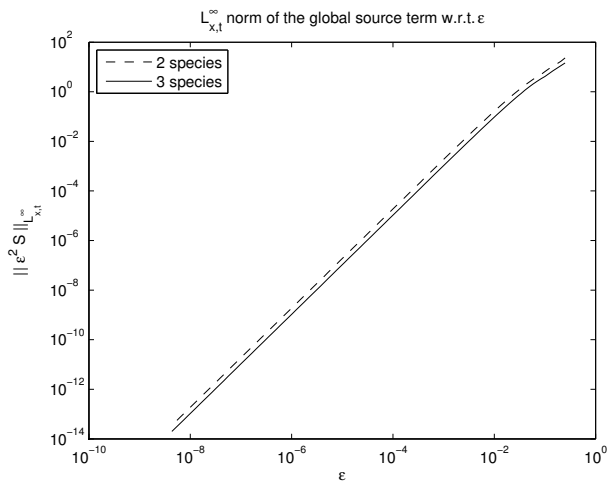

Figure 3. Maximum $M_{\varepsilon}$ of the source terms with respect to $\varepsilon$

\subsection{Three-species diffusion.}

We focus now on the three-species case, which shows a much richer behavior. Consider the following initial conditions

$$
c_{1}^{\text {in }}(x)=0.8 \times \mathbb{1}_{[-1,0]}, \quad c_{2}^{\text {in }}(x)=0.2, \quad c_{3}^{\text {in }}(x)=0.8 \times \mathbb{1}_{[0,1]}, \quad F_{1}^{\text {in }}(x)=F_{2}^{\text {in }}(x)=F_{3}^{\text {in }}(x)=0 .
$$

5.3.1. Uphill diffusion rising. Duncan and Toor's experiment [18] shows that this configuration enables the so-called uphill diffusion phenomenon, meaning that nitrogen, although being already at equilibrium, moves because of the movement of other species and their particular friction properties, due to their different mass ratios. This behavior is indeed observed, for $\varepsilon=10^{-2}$, as shown in Figure 4. After some time (corresponding to the diffusion barrier), the classical diffusion takes over and all species diffuse towards equilibrium.

As for the two-species case, we first check the consistency with the closure relation $c=1$ (see Figure 2), and the assumption on the source terms $\left(M_{\varepsilon}\right.$ tends to zero when $\varepsilon$ tends to zero, see Figure 3 .

5.3.2. Asymptotic behavior of the scheme and convergence towards the Maxwell-Stefan equations. The numerical scheme described in this paper has been especially designed in order to nicely degenerate for arbitrary small values of $\varepsilon$. Moreover, it has been proved, at least formally [11, 9], that the Boltzmann equations for mixtures tend to the Maxwell-Stefan equations when $\varepsilon$ vanishes. Therefore, for each species, we compute the $L^{\infty}$-norm in both $x$ and $t$ of the difference between its concentration and the concentration computed by Maxwell-Stefan equations. The computation of the solution to the Maxwell-Stefan equations is done as in [10], in particular using the closure relation corresponding to equimolar diffusion (i.e. the total flux of the mixture is identically equal to 0$)$. This is done for several values of $\varepsilon$, and shown on Figure 5 . The apparent order of convergence of the scheme is better than 1.

We observe the expected behavior: the smaller $\varepsilon$ is, the closer to Maxwell-Stefan equations we are. Let us emphasize that these results are obtained for fixed $\Delta x$ and $\Delta t$, as given at the beginning of the section. Therefore, the scheme does not need any restrictive condition on the parameters in order to be accurate for small values of $\varepsilon$. 

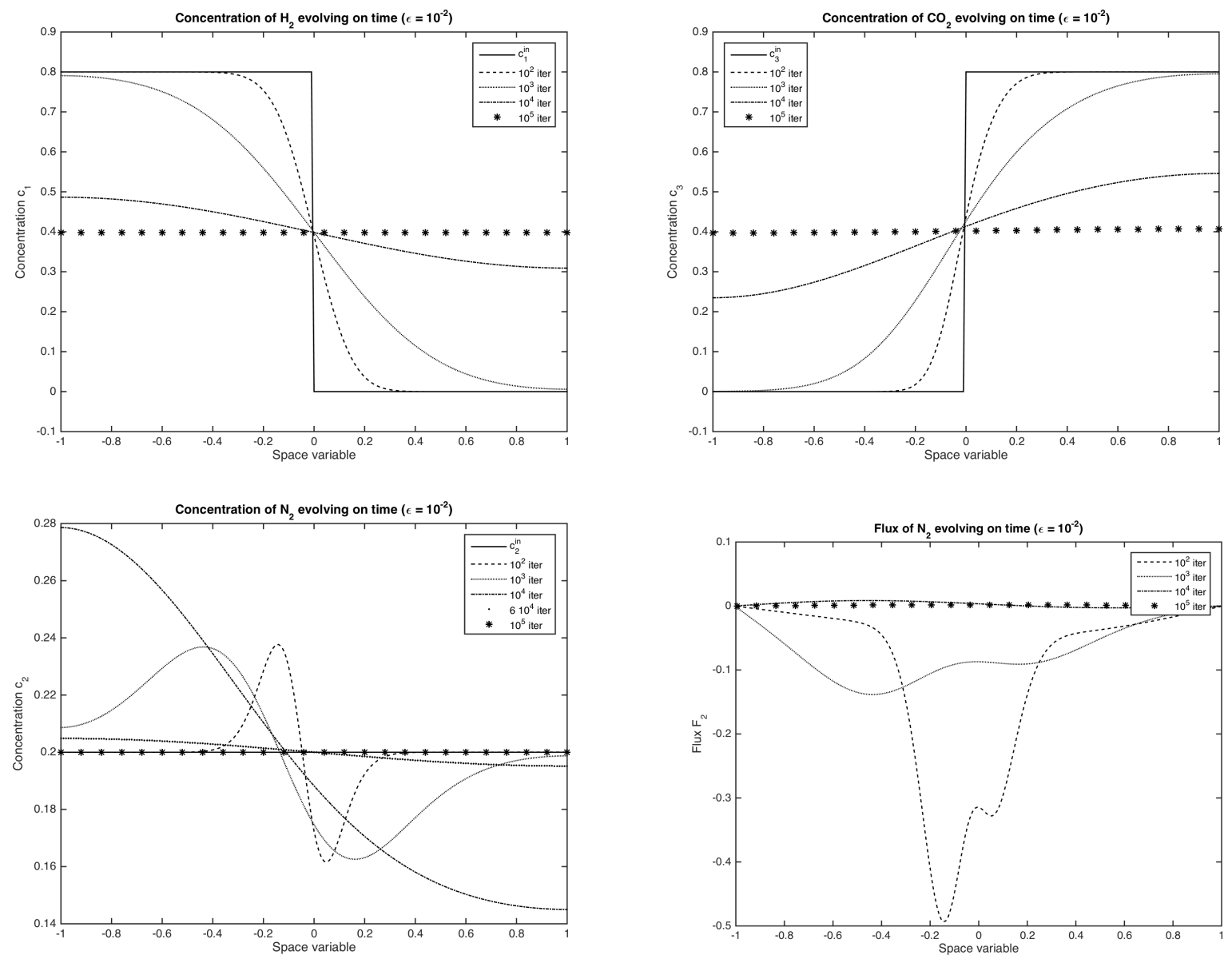

Figure 4. Evolution of the concentrations of species 1, 2 and 3, as well as the flux of species 2 in time in the three-species test case

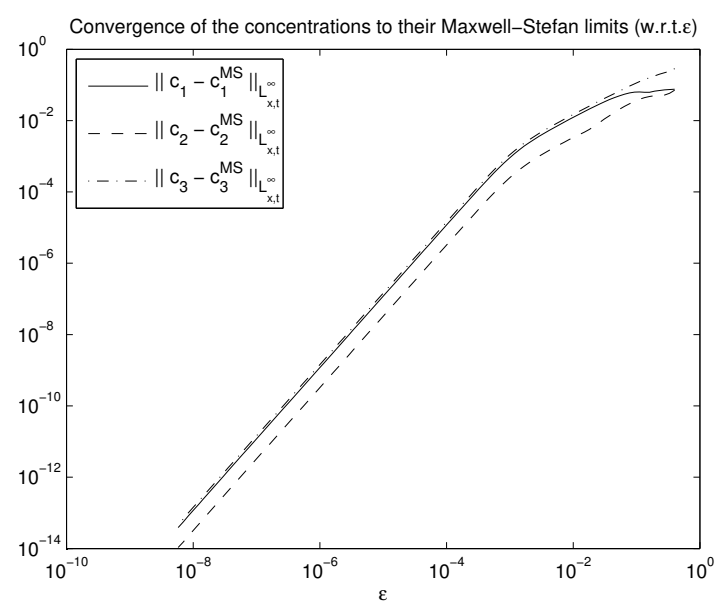

FiguRE 5. $\quad L_{x, t}^{\infty}$-norm of the difference between the computed concentrations and the solutions of Maxwell-Stefan equations for species 1, 2 and 3 with respect to $\varepsilon$ 

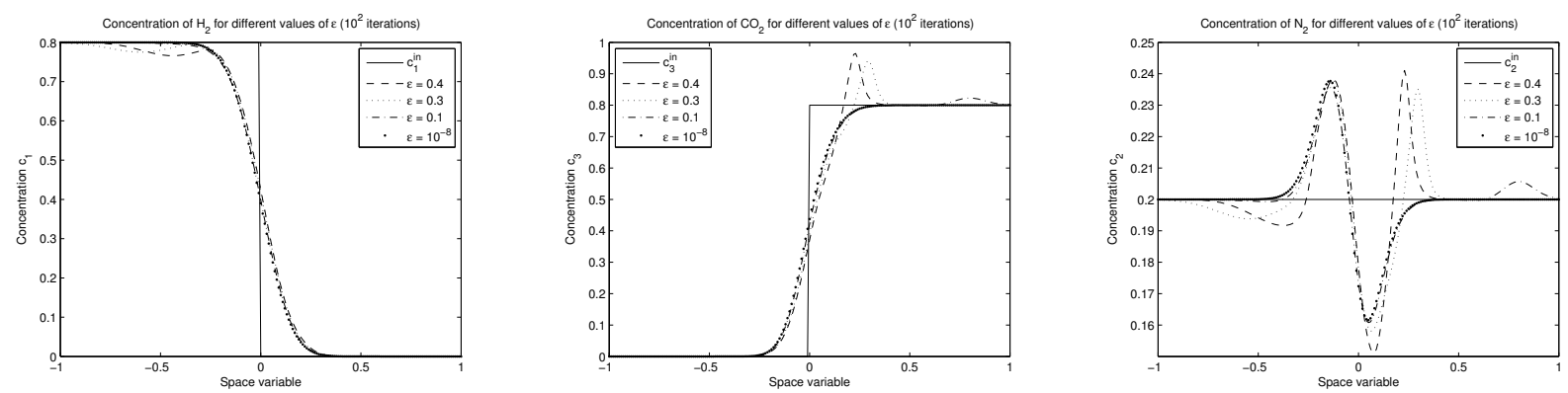

Figure 6. Concentrations of species 1,2 and 3 at time $t=10^{-2}$ with respect to $\varepsilon$

5.3.3. Influence of the parameter $\varepsilon$ on the diffusion process. We can also observe the influence of $\varepsilon$ on the diffusion process (see Figure 6).

As we already observed, for small values of $\varepsilon$, the diffusion is very similar to the Maxwell-Stefan's one. However, for larger values of $\varepsilon$ we observe some variations in the diffusion process. Although the diffusion process is quite different, the concentrations still converge in time to the same equilibrium as for smaller values of $\varepsilon$.

5.3.4. Velocity distributions. As stated before, the numerical scheme presented here relies on the moment method to treat the kinetic equation (7). More precisely, by using ansatz (8) stating that the distribution functions are at local Maxwellian states, we compute the zero-th and first order moments in velocity of $f_{i}^{\varepsilon}(t, x, v)$. On Figure 7, we plot the distribution function $f_{1}^{\varepsilon}(t, x, v)$ with respect to $v$ for $\varepsilon=10^{-2}, x=-0.21$ and several times $t$ (same values $t=0, t=10^{-2}, t=10^{-1}$, $t=1$ and $t=10$ as before). The amplitude of the Maxwellian is of course decreasing, since $c_{1}(t, x)$ decreases with time at the chosen value of $x$. In our configuration, the fluxes remain of order $10^{-1}$, which corresponds to velocities $u_{i}$ of order 1 (see Figure 4). Therefore, the Maxwellian are centered around $\varepsilon u_{1} \simeq 10^{-2}$, and the shift cannot be seen on the plot.

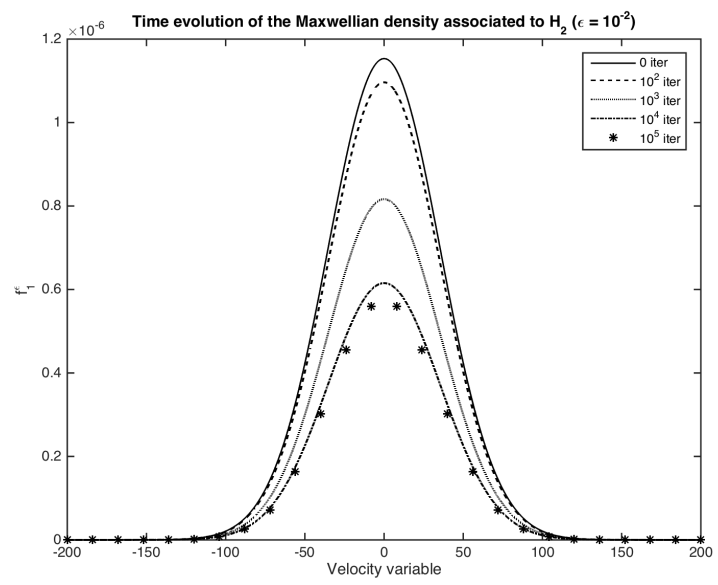

Figure 7. Evolution of the velocity distribution of species 1 at $x=-0.21$ in the three-species case

\section{REFERENCES}

[1] V. Anaya, M. Bendahmane, and M. Sepúlveda. Numerical analysis for a three interacting species model with nonlocal and cross diffusion. ESAIM Math. Model. Numer. Anal., 49(1):171-192, 2015. 
[2] P. Andries, K. Aoki, and B. Perthame. A consistent BGK-type model for gas mixtures. J. Statist. Phys., 106(56):993-1018, 2002.

[3] C. Bardos, F. Golse, and C. D. Levermore. Fluid dynamic limits of kinetic equations. I. Formal derivations. $J$. Statist. Phys., 63(1-2):323-344, 1991.

[4] C. Bardos, F. Golse, and C. D. Levermore. Fluid dynamic limits of kinetic equations. II. Convergence proofs for the Boltzmann equation. Comm. Pure Appl. Math., 46(5):667-753, 1993.

[5] S. Bastea, R. Esposito, J. L. Lebowitz, and R. Marra. Binary fluids with long range segregating interaction. I. Derivation of kinetic and hydrodynamic equations. J. Statist. Phys., 101(5-6):1087-1136, 2000.

[6] C. Bianca and C. Dogbe. Recovering Navier-Stokes equations from asymptotic limits of the Boltzmann gas mixture equation. Commun. Theor. Phys., 65:553-562, 2016.

[7] M. Bisi and L. Desvillettes. Formal passage from kinetic theory to incompressible Navier-Stokes equations for a mixture of gases. ESAIM Math. Model. Numer. Anal., 48(4):1171-1197, 2014.

[8] D. Bothe. On the Maxwell-Stefan approach to multicomponent diffusion. In Parabolic problems, volume 80 of Progr. Nonlinear Differential Equations Appl., pages 81-93. Birkhäuser/Springer Basel AG, Basel, 2011.

[9] L. Boudin, B. Grec, and V. Pavan. The Maxwell-Stefan diffusion limit for a kinetic model of mixtures with general cross sections. Nonlinear Anal., 159:40-61, 2017.

[10] L. Boudin, B. Grec, and F. Salvarani. A mathematical and numerical analysis of the Maxwell-Stefan diffusion equations. Discrete Contin. Dyn. Syst. Ser. B, 17(5):1427-1440, 2012.

[11] L. Boudin, B. Grec, and F. Salvarani. The Maxwell-Stefan diffusion limit for a kinetic model of mixtures. Acta Appl. Math., 136:79-90, 2015.

[12] S. Brull, V. Pavan, and J. Schneider. Derivation of a BGK model for mixtures. Eur. J. Mech. B Fluids, 33:74-86, 2012.

[13] L. Chen and A. Jüngel. Analysis of a multidimensional parabolic population model with strong cross-diffusion. SIAM J. Math. Anal., 36(1):301-322, 2004.

[14] A. De Masi, R. Esposito, and J. L. Lebowitz. Incompressible Navier-Stokes and Euler limits of the Boltzmann equation. Comm. Pure Appl. Math., 42(8):1189-1214, 1989.

[15] L. Desvillettes, T. Lepoutre, and A. Moussa. Entropy, duality, and cross diffusion. SIAM J. Math. Anal., 46(1):820-853, 2014.

[16] L. Desvillettes, T. Lepoutre, A. Moussa, and A. Trescases. On the entropic structure of reaction-cross diffusion systems. Comm. Partial Differential Equations, 40(9):1705-1747, 2015.

[17] L. Desvillettes, R. Monaco, and F. Salvarani. A kinetic model allowing to obtain the energy law of polytropic gases in the presence of chemical reactions. Eur. J. Mech. B Fluids, 24(2):219-236, 2005.

[18] J. B. Duncan and H. L. Toor. An experimental study of three component gas diffusion. AIChE Journal, 8(1):3841, 1962.

[19] L. C. Evans. Partial differential equations, volume 19 of Graduate Studies in Mathematics. American Mathematical Society, Providence, RI, 1998.

[20] A. Fick. Ueber Diffusion. Ann. der Physik, 170:59-86, 1855.

[21] F. Filbet and S. Jin. A class of asymptotic-preserving schemes for kinetic equations and related problems with stiff sources. J. Comput. Phys., 229(20):7625-7648, 2010.

[22] V. Garzó, A. Santos, and J. J. Brey. A kinetic model for a multicomponent gas. Phys. Fluids A, 1(2):380-383, 1989.

[23] V. Giovangigli. Convergent iterative methods for multicomponent diffusion. Impact Comput. Sci. Engrg., 3(3):244-276, 1991.

[24] V. Giovangigli. Multicomponent flow modeling. Modeling and Simulation in Science, Engineering and Technology. Birkhäuser Boston, Inc., Boston, MA, 1999.

[25] F. Golse and L. Saint-Raymond. The Navier-Stokes limit of the Boltzmann equation for bounded collision kernels. Invent. Math., 155(1):81-161, 2004.

[26] F. Golse and L. Saint-Raymond. The incompressible Navier-Stokes limit of the Boltzmann equation for hard cutoff potentials. J. Math. Pures Appl. (9), 91(5):508-552, 2009.

[27] F. Huang, Y. Wang, and T. Yang. Fluid dynamic limit to the Riemann solutions of Euler equations: I. superposition of rarefaction waves and contact discontinuity. Kinet. Relat. Models, 3:685-728, 2010.

[28] F. Huang, Y. Wang, and T. Yang. Hydrodynamic limit of the Boltzmann equation with contact discontinuities. Comm. Math. Phys., 295:293-326, 2010.

[29] H. Hutridurga and F. Salvarani. Maxwell-Stefan diffusion asymptotics for gas mixtures in non-isothermal setting. Nonlinear Anal., 159:285-297, 2017.

[30] H. Hutridurga and F. Salvarani. On the Maxwell-Stefan diffusion limit for a mixture of monatomic gases. Math. Methods Appl. Sci., 40(3):803-813, 2017.

[31] S. Jin. Asymptotic preserving (AP) schemes for multiscale kinetic and hyperbolic equations: a review. Riv. Math. Univ. Parma (N.S.), 3(2):177-216, 2012. 
[32] S. Jin and Q. Li. A BGK-penalization-based asymptotic-preserving scheme for the multispecies Boltzmann equation. Numer. Methods Partial Differential Equations, 29(3):1056-1080, 2013.

[33] S. Jin and Y. Shi. A micro-macro decomposition based asymptotic preserving scheme for the multispecies Boltzmann equation. SIAM J. Math. Anal., 31:4580-4606, 2010.

[34] A. Jüngel. The boundedness-by-entropy method for cross-diffusion systems. Nonlinearity, 28(6):1963-2001, 2015.

[35] A. Jüngel and I. V. Stelzer. Existence analysis of Maxwell-Stefan systems for multicomponent mixtures. SIAM J. Math. Anal., 45(4):2421-2440, 2013.

[36] R. Krishna and J. A. Wesselingh. The Maxwell-Stefan approach to mass transfer. Chem. Eng. Sci., 52:861-911, 1997.

[37] C. D. Levermore. Moment closure hierarchies for kinetic theories. J. Statist. Phys., 83(5-6):1021-1065, 1996.

[38] J. C. Maxwell. On the dynamical theory of gases. Phil. Trans. R. Soc., 157:49-88, 1866.

[39] C. Meyer. Matrix analysis and applied linear algebra. Society for Industrial and Applied Mathematics (SIAM), Philadelphia, PA, 2000.

[40] T. F. Morse. Kinetic model equations for a gas mixture. Phys. Fluids, 7:2012-2013, 1964.

[41] I. Müller and T. Ruggeri. Rational extended thermodynamics, volume 37 of Springer Tracts in Natural Philosophy. Springer-Verlag, New York, second edition, 1998. With supplementary chapters by H. Struchtrup and Wolf Weiss.

[42] R. J. Plemmons. M-matrix characterizations. I. Nonsingular M-matrices. Linear Algebra and Appl., 18(2):175188, 1977.

[43] L. Sirovich. Kinetic modeling of gas mixtures. Phys. Fluids, 5:908-918, 1962.

[44] J. Stefan. Ueber das Gleichgewicht und die Bewegung insbesondere die Diffusion von Gasgemengen. Akad. Wiss. Wien, 63:63-124, 1871.

[45] S.-H. Yu. Hydrodynamic limits with shock waves of the Boltzmann equation. Comm. Pure Appl. Math., 58:409443, 2005.

A.B., B.G.: MAP5, CNRS UMR 8145, Sorbonne Paris Cité, Université Paris Descartes, F-75006 Paris, FRANCE

E-mail address: andrea.bondesan@parisdescartes.fr

E-mail address: berenice.grec@parisdescartes.fr

L.B.: Sorbonne Université, Université Paris-Diderot SPC, CNRS, Inria, Laboratoire Jacques-Louis Lions, ÉQUipe Reo, F-75005 PARIS, France

E-mail address: laurent.boudin@sorbonne-universite.fr 